\title{
Time-resolved investigation of magnetization dynamics of arrays of nonellipsoidal nanomagnets with nonuniform ground states
}

\author{
P. S. Keatley, V. V. Kruglyak, A. Neudert, E. A. Galaktionov, and R. J. Hicken \\ School of Physics, University of Exeter, Stocker Road, Exeter, EX4 4QL, United Kingdom \\ J. R. Childress and J. A. Katine \\ Hitachi Global Storage Technologies, San Jose Research Center, 3403 Yerba Buena Road, San Jose, California 95135, USA
}

(Received 24 October 2007; published 9 December 2008)

\begin{abstract}
Time-resolved scanning Kerr microscopy measurements have been performed upon arrays of square ferromagnetic nanoelements of different sizes and for a range of bias fields. The experimental results were compared to micromagnetic simulations of model arrays in order to understand the nonuniform precessional dynamics within the elements. In the experimental spectra acquired from an element of length of $236 \mathrm{~nm}$ and thickness of $13.6 \mathrm{~nm}$, two branches of excited modes were observed to coexist above a particular bias field. Below this so-called crossover field, the higher frequency branch was observed to vanish. Micromagnetic simulations and Fourier imaging revealed that modes from the higher frequency branch had large amplitude at the center of the element where the effective field was parallel to the bias field and the static magnetization. Modes from the lower frequency branch had large amplitude near the edges of the element perpendicular to the bias field. The simulations revealed significant canting of the static magnetization and effective field away from the direction of the bias field in the edge regions. For the smallest element sizes and/or at low bias field values, the effective field was found to become antiparallel to the static magnetization. The simulations revealed that the majority of the modes were delocalized with finite amplitude throughout the element while the spatial character of a mode was found to be correlated with the spatial variation in the total effective field and the static magnetization state. The simulations also revealed that the frequencies of the edge modes are strongly affected by the spatial distribution of the static magnetization state both within an element and within its nearest neighbors. Furthermore, the simulations suggest that collective modes may be supported in arrays of interacting nanomagnets, which act as magnonic crystals.
\end{abstract}

DOI: 10.1103/PhysRevB.78.214412

PACS number(s): 75.40.Gb, 75.30.Ds, 75.50.Ss, 78.47.-p

\section{INTRODUCTION}

During the past decade the magnetization dynamics of thin-film magnetic materials have been studied intensively since these processes are expected to underlie the highfrequency operation of future recording head sensors, ${ }^{1}$ magnetic random access memory elements, ${ }^{2}$ and spin-torque devices. ${ }^{3}$ Experiments have been performed in which magnetization precession has been induced by harmonic ${ }^{4}$ or pulsed ${ }^{5}$ magnetic fields, ultrashort optical pulses, ${ }^{6}$ and a dc spin polarized current. ${ }^{7}$ In the majority of such studies, the samples were essentially unbounded and the ground-state magnetization was virtually uniform. Consequently, the precession frequency was fully determined by the magnetic parameters of the samples and the value of the applied (bias) magnetic field. ${ }^{8}$ In contrast, the internal magnetic field in finite sized nonellipsoidal magnetic elements is nonuniform, which introduces additional complexity into the character of the observed magnetization dynamics. For example, the nonuniform demagnetizing field may lead to the spatial confinement and quantization of spin-wave modes on the nanometer length scale. ${ }^{9-25}$ For thin-film elements where the magnetization lies in plane, the magnitude of the static in-plane demagnetizing field and the nonuniformity of the total effective field acting upon the magnetization increase when the element aspect ratio (size to thickness) is reduced. This results in a richer mode spectrum and hence in a less uniform magnetic response to a pulsed magnetic field, which can be di- rectly imaged in the case of micrometer sized magnetic elements..$^{26-32}$ The dominant role of the long-range magnetodipole interaction in the phenomena observed so far makes their analytical description complicated and generally requires numerical solution of integrodifferential equations. However, the interpretation of the magnetization dynamics in nonellipsoidal elements at finite bias field values becomes even more involved and challenging since it is not only the effective internal magnetic field but also the static magnetization that is nonuniform. This has led to the development and successful use of numerical algorithms for the simulation of the spatial dependence and temporal evolution of the magnetization dynamics in thin-film magnetic elements. ${ }^{33}$

In our previous study of 2.5 -nm-thick square nanomagnets of less than $200 \mathrm{~nm}$ size, we observed a dynamical regime in which the response to a uniform pulsed magnetic field was dominated by nonuniform precessional modes localized near the edges perpendicular to the direction of the bias field. ${ }^{15,16}$ Micromagnetic simulations of magnetization dynamics in thicker elements revealed the possible existence of a greater number of modes of a more complicated character, ${ }^{19}$ and also suggested that the exact ground state of the magnetization in the nanoelements might be the key factor in determining their dynamical properties.

In order to explore the effect of the magnetic ground state upon the mode spectrum of nanoelements in more detail, we now present measurements on a set of samples that are similar in composition, shape, and lateral size to those in Refs. 15 
and 16 but have about five times greater total thickness, and hence are characterized by a greater nonuniformity of the static magnetization and the total effective field within the element. We found that the precessional mode spectra vary in a discontinuous and complicated fashion as the bias magnetic field is reduced. In order to understand the evolution of the spectra, micromagnetic simulations were performed. It was found that the best agreement between the experimental spectra and those obtained from micromagnetic simulations was achieved when the following features were included in the simulations. First, imperfections of element shape were included in the simulations. Second, model arrays of $3 \times 3$ elements were studied so that the magnetic environment of the center element is correctly modeled. Third, the static magnetization state of all elements in the model array was carefully prepared by applying a similar bias field history to the model arrays as in the experiment. All three factors were found to be important in reproducing the experimental spectra and ultimately understanding the evolution of the spatial character of modes excited in elements of different sizes and at different values of the bias field.

Despite a reasonable agreement between the experimental and simulated spectra, the assignment of the mode character becomes far less clear than in the case of the thinner elements in Ref. 16. In particular, we found that the observed behavior evokes that of spin-wave well modes observed in larger $3 \mu \mathrm{m}$ square Permalloy elements presented in Ref. 17. In Ref. 17 the authors show that a combination of both the equilibrium and in-plane dynamic dipolar internal fields give rise to the localization of modes in the transition region between high and low internal fields, away from which the mode amplitude decreases. A general feature of the results presented in this paper is the occurrence of a crossover from a quasiuniform excitation that occupies the majority of the element volume to a regime that is dominated by several modes weakly localized near the element edges that lie perpendicular to the bias field. The transition was found to be mediated by the increasingly nonuniform static magnetization at low bias field values and by the associated nonuniformity of the total effective field throughout the element. There have been many reports on the excitation of similar modes in micrometer sized elements. ${ }^{11,12,14,17,20}$ In Ref. 24 the evolution of the mode character was studied for noninteracting nanoscale elements in which the static magnetization was assumed to be uniform. However, the evolution of such dynamics in arrays of interacting nanoscale elements as the bias field is reduced below $1 \mathrm{kOe}$ to remanence, for which both the static magnetization and internal field become nonuniform, have not previously been reported. Furthermore, we found that the frequencies of modes belonging to the lower frequency branch are very sensitive to the nonuniform ground state. Our experimental spectra also reveal that the lower frequency branch exhibits significant linewidth broadening, particularly at low bias fields and/or small element sizes.

The increased thickness, the associated enhanced stray magnetodipole field, and more nonuniform magnetization are also known to result in a stronger interaction of magnetic elements within arrays. ${ }^{10,18}$ The interaction has manifested itself before as extrinsic configurational anisotropy ${ }^{10}$ and as correlated switching of individual nanoelements within arrays. ${ }^{34}$ It has also been shown to lead to the splitting of normal modes of individual circular elements of $200 \mathrm{~nm}$ diameter within an array with a $50 \mathrm{~nm}$ edge-to-edge separation, ${ }^{18}$ which has been interpreted as the formation of collective spin-wave modes of the arrays. ${ }^{35}$ Our simulations on $3 \times 3$ element arrays suggest that the finite-size $(\sim 4$ $\times 4 \mu^{2}$ ) experimental arrays may also support several collective excitations with different frequency. The collective excitations are expected at lower bias field values for which the magnetostatic interaction between elements is enhanced as a result of the nonuniform magnetization. The particular boundary conditions at the edges of the arrays dictate the character of the collective excitations. Experimentally it might not be possible to resolve the collective modes, which may then appear as lower frequency modes of increased linewidth in our experiments.

\section{METHODS}

\section{A. Experimental technique}

For the measurements presented here, we used the same time-resolved scanning Kerr microscope (TRSKM) and methodology as in Ref. 16. Square $\sim 4 \times 4 \mu \mathrm{m}^{2}$ arrays of square elements of length (separation) of 637 (25), 428 (17), 236 (77), 124 (30), and 70 (37) nm were studied. The arrays were formed from a $\mathrm{Ta}(50 \AA) / \mathrm{Co}_{80} \mathrm{Fe}_{20}(40 \AA) /$ $\mathrm{Ni}_{88} \mathrm{Fe}_{12}(108 \AA) / \mathrm{Ta}(100 \AA)$ film sputtered onto a Si wafer, and patterned by electron-beam lithography and ion milling. Uniaxial anisotropy was induced in the sheet material by field annealing prior to the postdeposition array fabrication. In order to perform pump-probe measurements, two 300-nmthick $\mathrm{Au}$ transmission lines with width and separation of $30 \mu \mathrm{m}$ were fabricated on either side of the arrays parallel to the field annealing direction. A GaAs (substrate)/Au (300 $\mathrm{nm}$ ) photoconductive switch was connected to one end of the transmission lines while a bias voltage of $\sim 20 \mathrm{~V}$ was applied to the other end. Optical gating of the photoconductive switch using the pump-laser pulse caused a current pulse to propagate along the transmission lines, which in turn generated a pulsed magnetic field around the transmission lines. The pump-probe experiments were performed at a wavelength of $785 \mathrm{~nm}$. The probe beam was linearly polarized and then expanded by a factor of 10 to reduce the beam divergence. A microscope objective of numerical aperture of 0.65 was used to focus the probe beam to a diffraction limited spot with a submicrometer diameter. A piezoelectric scanning stage was used to position the center of the array under the probe spot. In this configuration, the measured polar Kerr rotation was proportional to the change in the out-of-plane component of the dynamic magnetization. The measured signal was an average response from an ensemble of elements at the center of the array within the probed region. The amplitude of the measured Kerr rotation was typically a few tens of microdegrees. A static bias magnetic field was applied in the plane of the sample parallel to the field annealing direction and the tracks of the stripline structure. The bias field was set by reducing the field from a value of $1.5 \mathrm{kOe}$ that far exceeded the saturation field observed in 
in-plane hysteresis loops acquired from these arrays using vector Kerr magnetometry. ${ }^{36}$

A continuous film reference sample was codeposited onto a 1-in.-diameter glass substrate. Vibrating sample magnetometry (VSM) measurements made upon reference samples with different bilayer thicknesses revealed that $12.1 \AA$ of the $\mathrm{Ni}_{88} \mathrm{Fe}_{12}$ layer was lost due to interdiffusion with the Ta capping layer. ${ }^{16}$ From the VSM measurements the static magnetizations of the $\mathrm{Co}_{80} \mathrm{Fe}_{20}$ and $\mathrm{Ni}_{88} \mathrm{Fe}_{12}$ were deduced to be 1445 and $585 \mathrm{emu} / \mathrm{cm}^{3}$, respectively. As the layers were strongly exchanged coupled, the bilayer was considered as a single layer with thickness of $13.6 \mathrm{~nm}$, which is equal to the sum of the thicknesses of the constituent layers after accounting for the effect of the interdiffusion. The magnetization of the bilayer was $838 \mathrm{emu} / \mathrm{cm}^{3}$ and was taken to be the volume-weighted average of the individual layer saturation magnetizations. Similarly to Ref. 16, time-resolved measurements made upon a codeposited $10 \mu \mathrm{m}$ square allowed the uniaxial $\left(9450 \pm 677 \mathrm{ergs} / \mathrm{cm}^{3}\right)$ and surface ( $-0.491 \pm 0.018 \mathrm{ergs} / \mathrm{cm}^{2}$ ) anisotropy parameters to be extracted by fitting the data to a macrospin model in the quasialignment approximation, and assuming a value of 2.1 for the $g$ factor. The easy axis was found to be canted by about $4^{\circ}$ from the direction of the striplines. The material parameters deduced here were used in micromagnetic simulations, which are described later. The validity of the assumption of quasialignment was verified by performing dynamical macrospin simulations in which the ground state or static orientation of the magnetization was determined using the steepest-descent method.

An optical micrograph obtained by scanning the sample beneath the focused laser beam is shown in Fig. 1(a). The different grayscale intensity of the different arrays corresponds to different packing fractions. Figure 1(b) shows the calculated profile of the in-plane and out-of-plane components of the pulsed magnetic field. ${ }^{37}$ Assuming a current of $64 \mathrm{~mA}$ of uniform density in the cross section of the striplines, the calculation revealed that the out-of-plane pulsed magnetic field was uniform to within $0.4 \%$ over the area of the array. Figure 1(c) shows a scanning electron microscope (SEM) image of the array of $236 \mathrm{~nm}$ square elements with a packing fraction of 56\%. From the image, one can see that the elements have slightly rounded corners. The SEM images of the studied elements were used to determine the radius of rounding of their corners and the interelement separations. Figure $1(\mathrm{~d})$ shows a $3 \times 3$ model array that corresponds to the SEM image in Fig. 1(c) and represents one of those used in the micromagnetic simulations. The inset of Fig. 1(a) shows the coordinate system used in the analysis and the direction of the bias field $\boldsymbol{H}$.

\section{B. Numerical simulations}

Micromagnetic simulations have been performed for all samples studied experimentally using the object oriented micromagnetic framework (OOMMF). ${ }^{38}$ Particular attention has been given to imperfections in the shape, magnetic environment, and ground state of the elements. Magnetostatic dipolar interactions with the nearest-neighboring elements
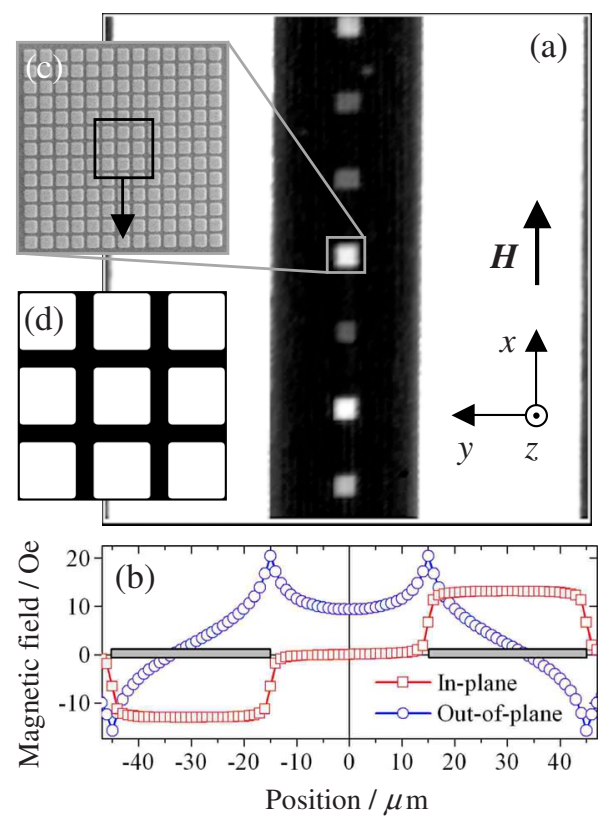

FIG. 1. (Color online) (a) An optical micrograph of the sample is shown. (b) The calculated profile of the in-plane (open squares) and out-of-plane (open circles) components of the pulsed magnetic field is shown. (c) A SEM image of the array of $236 \mathrm{~nm}$ square elements is shown. (d) A $3 \times 3$ model array corresponding to the SEM image in (c) and used in micromagnetic simulations is shown. The inset in (a) shows the coordinate system used in the paper.

are more significant in the thicker $(13.6 \mathrm{~nm})$ elements than for the previously studied ${ }^{16}$ thinner $(2.5 \mathrm{~nm})$ elements due to the increased magnetic charge on the element edges perpendicular to the bias field. To account for any modifications to the element ground state and splitting of the excited modes due to interelement interactions, $3 \times 3$ model arrays of square elements were generated. Details of element size, edge-toedge separation, element imperfection, and the radius of rounding of element corners were determined from the SEM images and were used to construct the model arrays used in the simulations. The radius of rounding of element corners was found to be $15 \mathrm{~nm}$ for all element sizes.

For each bias field two stages of numerical simulation were performed. In the first stage the ground state of the magnetization was calculated for which the energy of the magnetic system was minimized. The magnetization was relaxed quickly by setting the damping constant of the simulated material to 0.5 . To acquire the static state of the magnetization, two methods were investigated. In the first method the static state at each bias field was prepared by allowing the magnetization to relax from the uniform state. In this method the bias field was applied along the $x$ direction. In the second method, for the bias field of 1 kOe only, the static state was also prepared by relaxing the magnetization from the uniform state. However, subsequent static states at bias field values that are less than $1 \mathrm{kOe}$ were then prepared by relaxing the magnetization from the static state obtained at the previous bias field value. In the second method the bias field was applied $4^{\circ}$ from the $x$ direction, parallel to the uniaxial easy axis, so that all elements within the model array would have the same static magnetization state. 


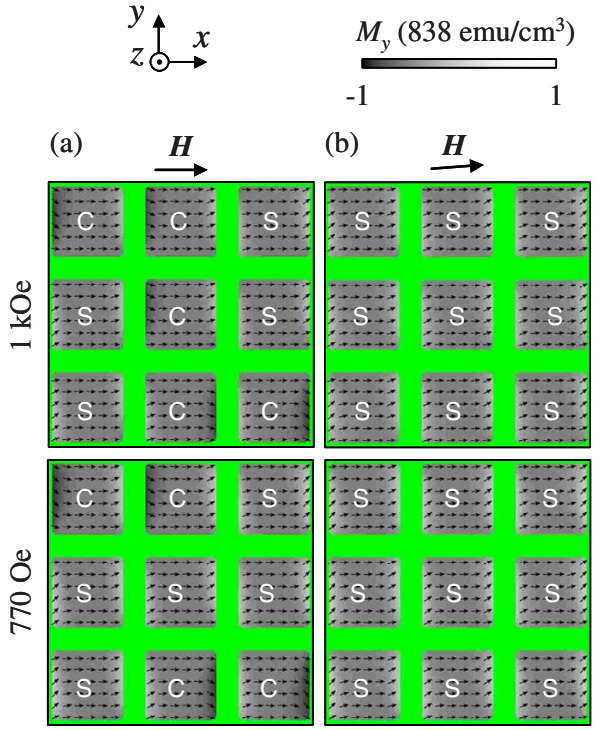

FIG. 2. (Color online) The static magnetization states of $3 \times 3$ model arrays of $236 \mathrm{~nm}$ elements simulated for bias field values of $1 \mathrm{kOe}$ and 770 Oe. The grayscale represents the component of magnetization that is parallel to the $y$ direction $\left(M_{y}\right)$ normalized to the saturation magnetization of $838 \mathrm{emu} / \mathrm{cm}^{3}$. The white and black shades represent $+M_{y}$ and $-M_{y}$, respectively. In (a) the static state at each bias field (applied parallel to the $x$ axis) was prepared by allowing the magnetization to relax from the uniform state. In (b) the static state at a bias field of $1 \mathrm{kOe}$ (applied $4^{\circ}$ from the $x$ axis) was prepared by relaxing the magnetization from the uniform state. Subsequently, the static state at 770 Oe was prepared by relaxing the magnetization from the state at $1 \mathrm{kOe}$.

It is known that OOMMF favors the flower ${ }^{39}$ or $X$ state due to the assumption of zero temperature. ${ }^{20}$ However, the $S$ state $^{39}$ can be stabilized at finite temperatures. ${ }^{20}$ We have also found that rounding of the element corners can promote the $C$ state $^{39}$ in some elements [Fig. 2(a)]. Canting the bias field by $4^{\circ}$ in the static simulation stabilized the $S$ state in the majority of elements [Fig. 2(b)]. When a bias field of $1 \mathrm{kOe}$ was canted from the $x$ direction by an angle of less than $4^{\circ}$, it was found that different elements within the array could acquire different static magnetization states. In Fig. 2 the results of the simulated static state of the $3 \times 3$ model arrays are shown for bias field values of $1 \mathrm{kOe}$ and 770 Oe for the two methods previously outlined. Figure 2(a) shows the results of the first method, relaxing from the uniform state, while Fig. 2(b) shows results of the second method, relaxing from the static state obtained at the previous bias field value. It is clear from the images that the static states obtained using the two methods are different. In particular in Fig. 2(a), for a bias field of $1 \mathrm{kOe}$, five elements were in the $C$ state (including the center element) and four elements were in the $S$ state. At 770 Oe the ground states of the elements surrounding the center element were unchanged while the center element was now in the $S$ state. Furthermore, as the bias field was reduced from 770 to 590 Oe (not shown), the static state of a corner element was found to change from an $S$ to a $C$ state. Such irregular changes in the static state were seen for all element sizes, and make the interpretation of the mode spectra and spatial character very difficult. In Fig. 2(b) at bias fields of $1 \mathrm{kOe}$ and $770 \mathrm{Oe}$, canting the bias field $4^{\circ}$ from the $x$ direction has led to all elements occupying the $S$ state. It was expected that if all elements occupied the same static state, then the interpretation of the mode spectra and spatial character would be more readily understood. Hence, the second method was used to simulate the static state for all element sizes. In the experiments, the bias field was not intentionally canted with respect to the $x$ direction although one should expect some misalignment of a few degrees to occur.

To test the effect of the static magnetization state upon the simulated spectra, it was often necessary to induce the $C$ state in the elements, i.e., to be certain that the $C$ state was obtained for all elements within a $3 \times 3$ array, and not mixed $S$ and $C$ states as in Fig. 2(a). The $C$ state was induced by initially defining a Landau flux closure domain pattern in the individual elements in which the magnetization was uniform in each domain. The chirality of the Landau state was clockwise for all elements. The magnetization was then relaxed at remanence with the damping constant set to 0.5 , which resulted in a single domain vortex structure within each element with the same chirality as the initially defined Landau state. The elements were then magnetized in steps of 5 Oe to a field of $1 \mathrm{kOe}$ applied in the positive $x$ direction. The vortex core was found to move in the negative $y$ direction before being eliminated from the element, which resulted in the magnetization occupying the $C$ state. The elements were magnetized further until the $X$ state was achieved. At a particular field value, the corresponding $C$ state was then acquired by relaxing the magnetization from the $C$ state obtained prior to the $X$-state transition.

The second stage of the micromagnetic simulations was to calculate the dynamic response of the magnetization of the model arrays. The damping parameter and $g$ factor were assumed to have values of 0.01 and 2.1 , respectively. As in the experiment, a uniform out-of-plane pulsed magnetic field was used to excite the magnetization precession. The pulsed magnetic field used in the simulations was assumed to have a rise time of $40 \mathrm{ps}$, a decay time constant of $2 \mathrm{~ns}$, and a magnitude of 15 Oe. ${ }^{16}$ All three Cartesian components of the dynamic magnetization were recorded at 20 ps intervals. The polar Kerr microscope used in the experiments was sensitive to the change in the out-of-plane component of the dynamic magnetization. Therefore, only the out-of-plane component recorded in the simulations was used in the analysis of the experimentally observed dynamics. Furthermore, the spot size of the optical probe is larger than any of the elements measured experimentally. For the center element within the model array, the nearest-neighbor environment was expected to be a good approximation to that of elements in the interior of the real arrays. Therefore, the average time-resolved response of only the center element was compared with that obtained experimentally. As in the experiment, the oscillation of the simulated out-of-plane component of the magnetization was found to be superimposed on a slowly varying background. The background was due to a transient canting of the magnetization which follows the temporal profile of the pulsed magnetic field. In the simulations, the form of the pulsed field is known exactly and can therefore be subtracted from the recorded traces of the averaged out-of-plane com- 


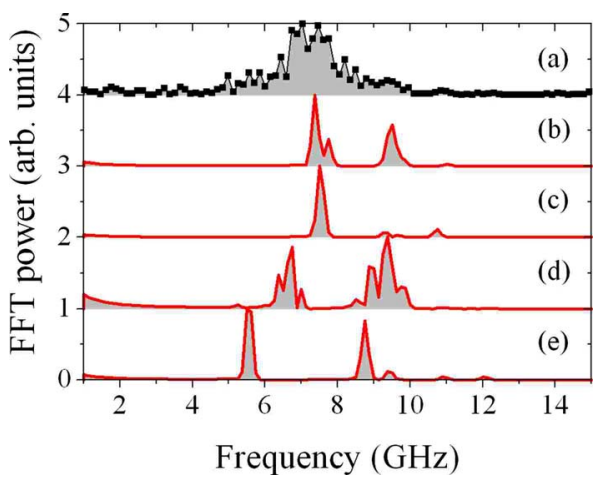

FIG. 3. (Color online) The (a) experimental and simulated spectra of (b) the center element of the $3 \times 3$ model array shown in Fig. 1(d), (c) a single element from that array, (d) the center element of a $3 \times 3$ array of perfect squares, and (e) a single perfect square are shown. The simulations were performed for a $236 \mathrm{~nm}$ element with a bias field of 590 Oe applied $4^{\circ}$ from the $x$ direction. The static magnetization of all the simulated elements was found to be in the $S$ state. It is clear from the simulated spectra that both imperfections in element shape and different magnetic environments give rise to very different spectra.

ponent of the dynamic magnetization in the center element.

To determine the spatial character of the excited modes, images of the modes were generated from the simulated time-resolved data as in Ref. 31. The magnetization at each time delay was recorded as a vector field map. For each pixel, a time-resolved trace was generated from the out-ofplane component of the magnetization. Fast Fourier transforms (FFTs) of the time-resolved trace for each pixel revealed the Fourier components that were excited in a particular pixel. The calculated FFTs were then used to reconstruct images of the magnitude and phase of the dynamical magnetization. Thus for each mode previously identified from the FFT spectra obtained from the average response of the center element, a corresponding "Fourier image" was calculated showing the spatial profile of the magnitude and phase of the dynamical magnetization. For clarity, the images of the FFT magnitude are normalized to $75 \%$ of the maximum value of the magnitude in the center element.

Prior to performing an extensive series of micromagnetic simulations, the model to be used in the simulation of the experimental spectra was investigated. The test simulations were performed for an element size of $236 \mathrm{~nm}$ at a bias field of 590 Oe applied $4^{\circ}$ from the $x$ direction. Figure 3 shows the (a) experimental and simulated spectra of (b) the center element of the $3 \times 3$ model array shown in Fig. 1(d), (c) a single element from that array, (d) the center element of a $3 \times 3$ array of perfect squares, and (e) a single perfect square. The static magnetization of all of the simulated elements was found to be in the $S$ state. The spectrum for the single perfect square element [Fig. 3(e)] yields poor agreement with the experimental spectrum with a lower frequency peak almost 2 $\mathrm{GHz}$ below the experimental peak. While the spectrum of the single element with rounded corners [Fig. 3(c)] was in better agreement with experiment, there was no splitting of the simulated peak to support the large linewidth observed experimentally. The spectrum of the rounded square array [Fig. 3(b) ] was found to have better agreement with the experimental spectra. In addition, reasonable agreement was found between the experimental spectrum and the spectrum of the perfect square array [Fig. 3(d)]. Therefore, we conclude that simulations using $3 \times 3$ model arrays are required to reproduce the experimental spectrum. Additional splitting of the higher and lower frequency modes was observed in the spectrum for the perfect square array. Such splitting was not observed in the array of elements with rounded corners and therefore might be an artifact of the sharp corners of the perfect elements. Also the relative weight of different modes within the spectrum of the rounded square array was found to match that of the experimental spectrum better than the spectrum of the perfect square array. Therefore, the array of elements with rounded corners was favored for simulations of the experimental spectra. The simulations in Fig. 3 also revealed that the frequencies of the higher frequency modes were less sensitive than those of the lower frequency modes to the edge conditions of the model, i.e., rounding of element corners and nearest-neighbor magnetostatic interactions. This was evident from a $\sim 2 \mathrm{GHz}$ variation in frequency of the lower frequency modes between the different models while the variation in frequency of the higher frequency mode was only $\sim 1 \mathrm{GHz}$. Indeed, Fourier images (to be presented and discussed later) reveal that the lower frequency modes are associated with edge regions of the element while the higher frequency modes are associated with the center region of the element, in agreement with the conclusions of Ref. 19.

The simulated spectra presented in the following sections of this paper are found to reproduce the general trends observed experimentally. However, they do not quantitatively reproduce all of the details of the experimental spectra since the simulated spectra are generated from the response of an individual element at the center of a $3 \times 3$ element array while experimentally, more than one element was probed. Furthermore the experimental spectra have a finite signal-tonoise ratio. In light of the agreement between the general trends observed in experimental and simulated spectra, we will discuss the results of the simulated Fourier images in order to gain some insight into the possible spatial character of the observed modes. Our aim is not to reproduce the exact structure of the experimental spectra using micromagnetic simulations but instead to interpret the general trends in the experimental spectra, for example, the crossover from highfrequency to low-frequency precession as the element size is reduced. Micromagnetic simulations have been used to explore in detail the sensitivity of the exact structure of the spectra to the element edge conditions, interelement interactions, and static magnetization state.

\section{RESULTS AND DISCUSSION}

\section{A. Time-resolved signals}

Figure 4(a) shows a typical time-resolved signal measured from the $236 \mathrm{~nm}$ element array at a bias field of 270 Oe. The oscillations due to the magnetization precession are superimposed on a slowly varying background due to a transient out-of-plane canting of the magnetization which follows the temporal profile of the pulsed magnetic field. The out-of- 

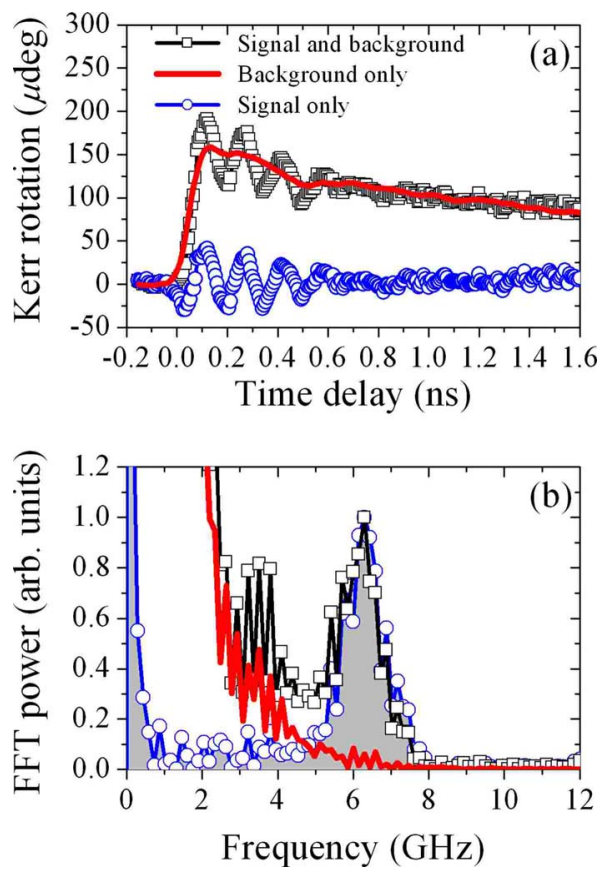

FIG. 4. (Color online) (a) A typical raw time-resolved signal (line with squares), the slowly varying background (solid line), and the signal with the background subtracted (line with circles) are shown for the array of $236 \mathrm{~nm}$ elements at a bias field of 270 Oe. (b) The FFT power spectra of the traces in (a) are shown.

plane canting of the magnetization gives rise to a transient polar Kerr signal onto which the oscillatory Kerr signal is superimposed. The temporal profile of the transient Kerr signal was determined from signals measured at a bias field of $\sim 1.5 \mathrm{kOe}$, at which no significant oscillatory response was observed. To isolate the oscillatory part of the signal, this background was subtracted from the time-resolved signals measured at lower field values. ${ }^{5}$ However, the form of the pulsed field background observed in the experiments was not known exactly. Therefore, the background cannot be completely removed which results in a large peak in the experimental spectra below $1 \mathrm{GHz}$. As mentioned in the previous section, the form of the pulsed field used in the dynamic simulations is known exactly, which allows the background to be completely removed. Figure 4(b) shows the FFT spectra calculated from the time-resolved signals in Fig. 4(a). The peaks in the spectra calculated from first the raw signal, and second the raw signal with the background subtracted, agree well for frequencies greater than about 5.5 GHz. The background subtraction extends the range of observable frequencies down to about $1 \mathrm{GHz}$.

In Fig. 4(b), the spectra are more noisy below the main spectral peak. The noise is related to the pulsed field background for which the corresponding spectrum in Fig. 4(b) reveals sharp peaks between 2 and $5 \mathrm{GHz}$. Since the exact form of the pulsed field background is not known, the peaks seen in the spectrum for the raw data cannot be removed completely. As a result the peaks associated with the pulsed field manifest themselves as noise in the spectrum of the time-resolved signal after the background has been sub-

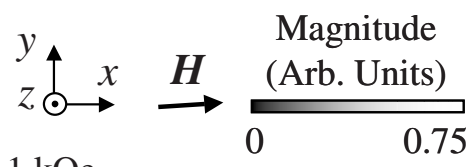

(a) $1 \mathrm{kOe}$

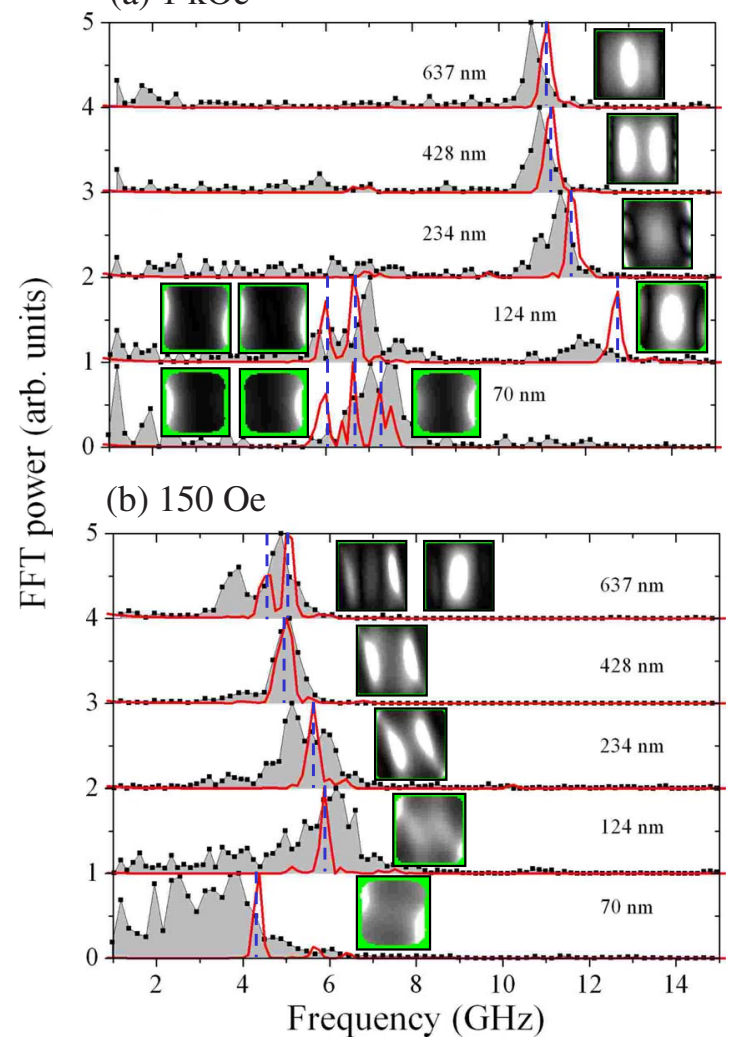

FIG. 5. (Color online) The dependence of the mode frequency upon the element size is shown for bias field values of (a) $1 \mathrm{kOe}$ and (b) 150 Oe. The shaded spectra were obtained experimentally while simulated spectra are shown with a solid curve. For each element size, the spatial distribution of the FFT magnitude of the modes in the simulated spectra is shown in the insets.

tracted, which then makes interpretation of precessional spectral peaks at lower frequencies more difficult.

\section{B. Size dependence}

We consider first the dependence of the mode character upon the element size. ${ }^{16}$ FFT spectra calculated from the measured and simulated time-resolved signals are shown in Fig. 5 for two values of the bias field (a) 1 kOe and (b) 150 Oe. At the bias field value of $1 \mathrm{kOe}$, the dependence of the experimental FFT spectra upon the size of the elements closely resembles that observed in Ref. 16. As the element size is reduced from 637 to $236 \mathrm{~nm}$, the frequency of the dominant mode initially increases. At an element size of 124 $\mathrm{nm}$, at least two modes are clearly seen in both the experimental and simulated data. The "higher frequency mode" continues the trend of increasing frequency as the element size is reduced; however in the $124 \mathrm{~nm}$ element, its spectral power is reduced. The "lower frequency mode" is separated from the higher frequency mode by about $5 \mathrm{GHz}$ in the experimental spectra and has slightly higher spectral power. 
The frequency of the lower frequency mode then increases as the element size is reduced further. The trend is reproduced by the simulated spectra. The spectra show a crossover in mode intensity for an element size of about $124 \mathrm{~nm}$ while for thinner elements $(2.5 \mathrm{~nm})$ in Ref. 16 the crossover was observed to occur at the larger element size of about $220 \mathrm{~nm}$. In Ref. 16, the crossover was interpreted in terms of the relative size of the demagnetized regions within elements with quasiuniform static magnetization. The size of the demagnetized regions is expected to increase as the thickness of the elements is increased and the aspect ratio is reduced. Therefore, one would expect the crossover to occur at a greater, rather than smaller, element size for the thicker elements in this study when compared to those of Ref. 16. However, exactly the opposite trend is observed in Fig. 5(a) with the crossover occurring at a smaller element size for the thicker elements. This suggests that the spatial distribution of the demagnetizing field is not the dominant factor that determines the character of the excited modes. The assumption of static uniform magnetization may also be incorrect. Indeed, the magnetization is expected to be less uniform in the thicker elements. Alternatively, the discrepancy may provide evidence for an increased magnetodipole interaction between elements as the elements become thicker.

As in Ref. 16, the simulated images of Fourier magnitude reveal that modes from the higher frequency branch occupy the majority of the element volume ("center modes") while the modes of the lower frequency branch are localized near the edges of the element that are perpendicular to the direction of the bias field ("edge modes"). All modes are nonuniform throughout the elements due to the localized nature of the modes, particularly for the edge modes of the lower frequency branch. Nonuniformity of the center modes from the higher frequency branch occurs in the direction of the bias field, as was previously observed in Ref. 19. In Ref. 19 a backward-volume-type mode was introduced since the effective wave vector is parallel to the magnetization. The nonuniformity arises because the center mode and backwardvolume-type mode have similar frequencies and finite linewidths, and therefore cannot be completely resolved. Also for the three largest elements, nonuniformity exists in the direction orthogonal to the bias field at the edges of the element that are perpendicular to the bias field.

At the bias field of 150 Oe, the behavior was remarkably different to that at $1 \mathrm{kOe}$. As the element size was reduced from 637 to $124 \mathrm{~nm}$, the frequency of the excited modes in the experimental spectra was observed to increase. When the element size was reduced further to $70 \mathrm{~nm}$, a shift of the spectral peak to lower frequency was observed, albeit with significant linewidth broadening. It is difficult to follow the evolution of a particular mode as the element size is reduced. This is likely to be due to slightly different static magnetization states of different element sizes resulting from different magnetostatic interactions from neighbors and different edge conditions, e.g., edge roughness. In general the experimental spectra at 150 Oe reveal broadening of the spectral peaks, which was found to be more significant for smaller element sizes. Inhomogeneous broadening due to edge roughness was expected to be enhanced for smaller elements, particularly at low bias fields for which the magnetization is nonuniform.
These random edge defects give rise to a distribution of pinning fields at the edges of the elements, which in turn give rise to a distribution of resonance frequencies and hence linewidth broadening. However, the simulated spectra were derived from the response of a single element at the center of $3 \times 3$ arrays. Therefore, significant broadening due to edge roughness was not expected in the simulated spectra. However, the simulations do reproduce the general trends observed in the experimental spectra, which allow us to use them to discuss the likely spatial character of the observed modes. The modes observed in the simulated Fourier images do not fall into the classification of center or edge mode used in Refs. 16 and 19, and in the previous paragraph. At 150 Oe, only the $637 \mathrm{~nm}$ element supports a center mode with spectral power that is greater than all other excited modes. However, a lower frequency mode was observed with spectral power of about $50 \%$ of that of the center mode. In the Fourier image of the lower frequency mode, the regions of high amplitude appear to be tilted relative to and detached from the element edges that lie perpendicular to the bias field. We will refer to these modes as "detached-edge modes." When the element size was reduced to $428 \mathrm{~nm}$, we see that the mode with greatest spectral power has a similar spatial character to the lower frequency mode of the $637 \mathrm{~nm}$ element. This is again the case in the $236 \mathrm{~nm}$ element. Thus at $150 \mathrm{Oe}$ we observed the size dependent crossover in mode spatial character between element sizes of 637 and $428 \mathrm{~nm}$. Comparison of the size dependent crossover in Fig. 5 at $1 \mathrm{kOe}$ and 150 Oe indicates a strong bias field dependence of this general feature of the dynamical behavior of nonellipsoidal nanomagnets. As the element size decreases we again see an increase in the frequency of the excited modes. However, at $124 \mathrm{~nm}$ the spatial character of the dominant mode appears to be somewhat different to that of the modes seen in the larger elements. We interpret the modified spatial profile of the mode to be the result of the two detached-edge modes (as seen in the element size of $236 \mathrm{~nm}$ ) merging at the center of the element. The dominant mode frequency is observed to be reduced by about $2 \mathrm{GHz}$ as the element size is reduced from 124 to $70 \mathrm{~nm}$, which might be indicative of a transition of the static magnetization state from an $S$ state to a leaf state. ${ }^{39}$ Consequently, the high amplitude regions have migrated to the edges of the element perpendicular to the bias field and slightly toward opposite diagonal corners where the effective field may be slightly reduced.

So far the modes shown in the Fourier images of Fig. 5 have been classified as center, edge, or detached-edge modes. However, it is not clear from Fig. 5 how the mode character evolved as the bias field was reduced from $1 \mathrm{kOe}$ to $150 \mathrm{Oe}$. In light of the clear field dependence of the mode spatial character, it is necessary to investigate and understand the evolution of the mode spatial character as the bias field is changed. Reducing the bias field for a particular element size allows one to study the effect of the evolution of the static magnetization, total effective magnetic field, and interelement dipolar coupling. The increased nonuniformity of the magnetic ground state in individual elements and/or the increased interaction between elements within the arrays will be shown to be important contributions to the evolution of mode spatial character. 


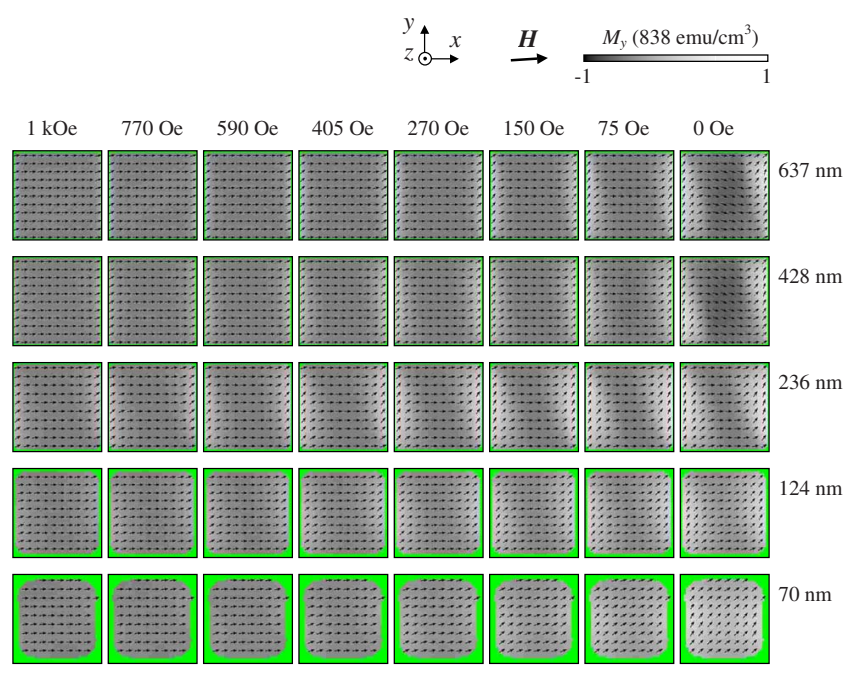

FIG. 6. (Color online) The simulated static magnetization states within the center element of $3 \times 3$ model arrays are shown. The grayscale represents $M_{y}$ normalized to $M_{s}=838 \mathrm{emu} / \mathrm{cm}^{3}$, where white and black represent $+M_{y}$ and $-M_{y}$, respectively. The bias field was applied $4^{\circ}$ from the $x$ direction in order to stabilize the $S$ state.

In order to understand the evolution of the spatial character, images of the ground-state magnetization have been simulated. Figure 6 shows images of the static state magnetization of the center element of the $3 \times 3$ model arrays for all element sizes and bias field values. The grayscale represents $M_{y}$ normalized to $838 \mathrm{emu} / \mathrm{cm}^{3}$, where white and black represent $+M_{y}$ and $-M_{y}$, respectively. At $1 \mathrm{kOe}$ and for all element sizes the magnetization was nearly saturated but exhibited features of the $S$ state where the magnetization near the edges of the elements perpendicular to the bias field was slightly canted toward the $y$ direction. Canting of the bias field $4^{\circ}$ from the $x$ direction has stabilized the $S$ state in the center element and the surrounding nearest-neighboring elements (not shown) for almost all element sizes and bias field values. At $150 \mathrm{Oe}$, all elements are still seen to be in the $S$ state. The images reveal that a transition from a nonuniform $S$ state to a similar but more uniform leaf state occurs between element sizes of 124 and $70 \mathrm{~nm}$, and bias fields of 75 and 0 Oe. Furthermore, for the $3 \times 3$ array of $70 \mathrm{~nm}$ elements, one of the corner elements acquires the $C$ state (not shown) for bias fields of 770 Oe and below. Further canting of the bias field to $5^{\circ}$ may eliminate this; however one corner element in the $C$ state has a minimal effect on the behavior of the center element so further simulations were not performed.

As in Ref. 16, the linewidth of the experimental spectra for the array of $70 \mathrm{~nm}$ elements at a bias field of $1 \mathrm{kOe}$ is noticeably greater than that in the larger elements. The simulations suggest that this may be due to several excited modes with frequencies between 5 and $8 \mathrm{GHz}$ that may not have been resolved in the experiment. In line with this interpretation, the linewidth of the experimental spectra measured at $150 \mathrm{Oe}$ is increased for all element sizes, which is echoed in the simulations by the excitation of multiple "satellite" peaks around the main spectral peak albeit with rather small spectral power. At the same time, failure to resolve the modes

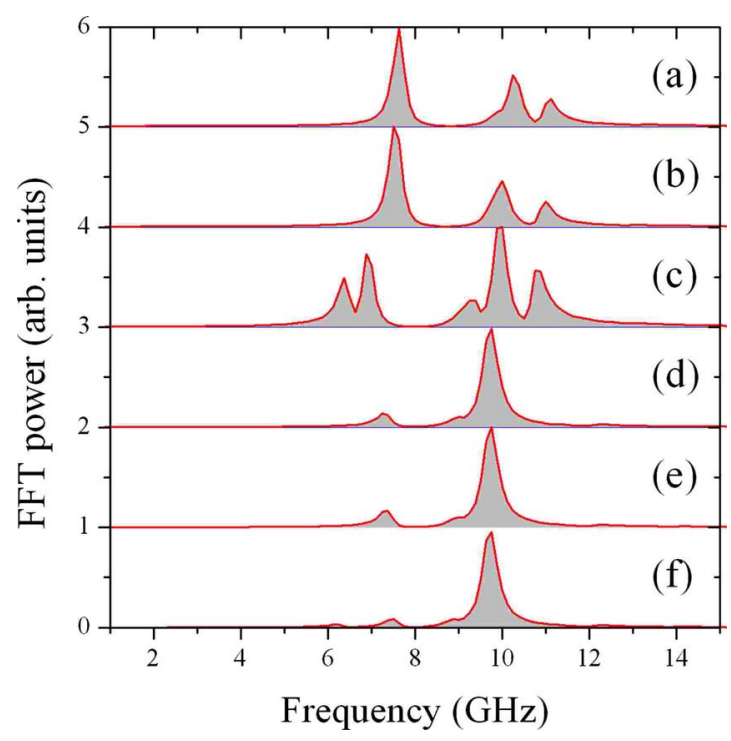

FIG. 7. (Color online) Simulated spectra for isolated $236 \mathrm{~nm}$ square elements with rounded corners are shown for (a) a square with no edge roughness, (b) a square with roughness along the edges of the element parallel to the bias field, and (c) a square with roughness along edges perpendicular to the bias field. The bias field strength was 770 Oe and was applied $4^{\circ}$ from the $x$ direction. The static magnetization of all the simulated elements was found to be in the $S$ state. In (d)-(f) the spectra are shown for the same elements as those in (a)-(c) but the magnetization occupies the $C$ state instead. Again the bias field was 770 Oe but was applied along the $x$ direction in order to prevent the $S$ state from being stabilized.

experimentally should be attributed to the random nature of edge defects and rounding of element corners that give rise to inhomogeneous broadening due to variations within the array. ${ }^{16}$ To verify this assumption, we performed micromagnetic simulations for nine isolated elements. All elements had different edge roughness, which was introduced by removing "magnetic" pixels from the edges of a nominally shaped square model element with rounded corners. The size and density of the structural defects was varied randomly; however the roughness was no more than $\sim 2.5 \mathrm{~nm}$ or $\sim 1 \%$ of the length of the nominal square shape. The nominal length of all nine elements was $236 \mathrm{~nm}$. A bias field of 590 Oe was applied $4^{\circ}$ from the $x$ direction for which the static magnetization of all elements was found to occupy the $S$ state. The simulations support the interpretation of the inhomogeneous broadening by revealing that a slight change in the shape of an element, due to different edge roughness, can cause the mode frequency to vary noticeably. This has also been reported in Ref. 25. In some cases the edge defects were found to result in the splitting of modes. Figure 5 suggests that modes localized near the edges in the elements of smaller sizes appear to be affected more. Two additional simulations were performed at 590 and 770 Oe for which defects were only included along edges of the element parallel to the bias field (case 1), and along edges of the element perpendicular to the bias field (case 2). The simulated spectra at 770 Oe are shown in Figs. 7(b) and 7(c), respectively, and are compared to the spectrum of an element with rounded corners and no defects, as shown in Fig. 7(a). In all three cases the rounding 


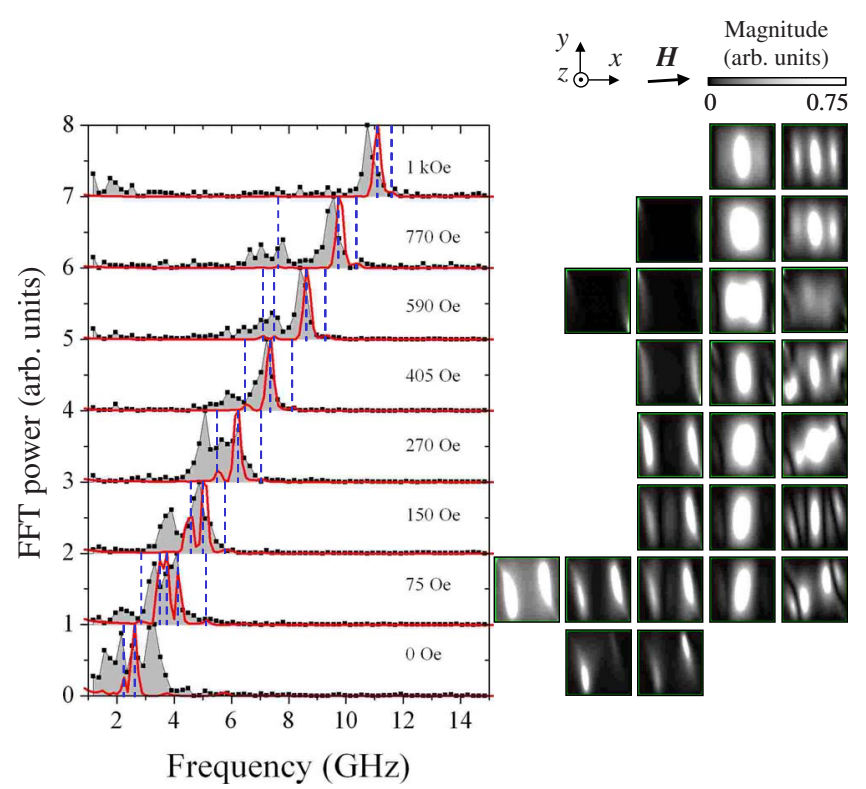

FIG. 8. (Color online) The dependence of the mode spectra and spatial character of the different modes upon the bias field is shown for the $637 \mathrm{~nm}$ element. The shaded spectra with symbols show the experimental data while the solid red line shows spectra obtained from micromagnetic simulations. The vertical dashed blue lines indicate the frequencies of the modes whose spatial character is shown in the images of the magnitude of the Fourier transform. The images are ordered in terms of increasing frequency.

of the element corners was identical. The spectra at each field value revealed that defects along the edges of the element perpendicular to the bias field (case 2) resulted in the largest variation in the mode frequencies. The variations seen in the spectra were most significant for the edge mode although additional shifting in frequency and splitting were seen for the center mode. Figure 7(c) shows that defects along the edges perpendicular to the bias field leads to splitting of the edge mode into two modes. Both of these modes have lower frequency than that of the lower frequency mode in the spectrum for the element with no defects [Fig. 7(a)]. In contrast, defects along the edges parallel to the bias field yield little variation in the spectrum [Fig. 7(b)]. It is also interesting to note that, for an isolated element in the $C$ state, edge roughness appears to have little effect on the spectra. Figures 7(d)-7(f) show the spectra for the same elements as those in Figs. 7(a)-7(c) but the magnetization occupied the $C$ state instead. As already mentioned, the simulated spectra presented later in this paper do not exhibit inhomogeneous broadening since the FFT spectra are calculated from the response of only the center element of the $3 \times 3$ arrays.

\section{Field Dependence}

In light of the strong dependence of the mode structure upon the exact shape of nanoelements, it is difficult to correlate the static and excited states of the magnetization through a comparison of elements of different sizes and hence slightly different shapes. On the other hand, the effective field and the static magnetization state within a particu-

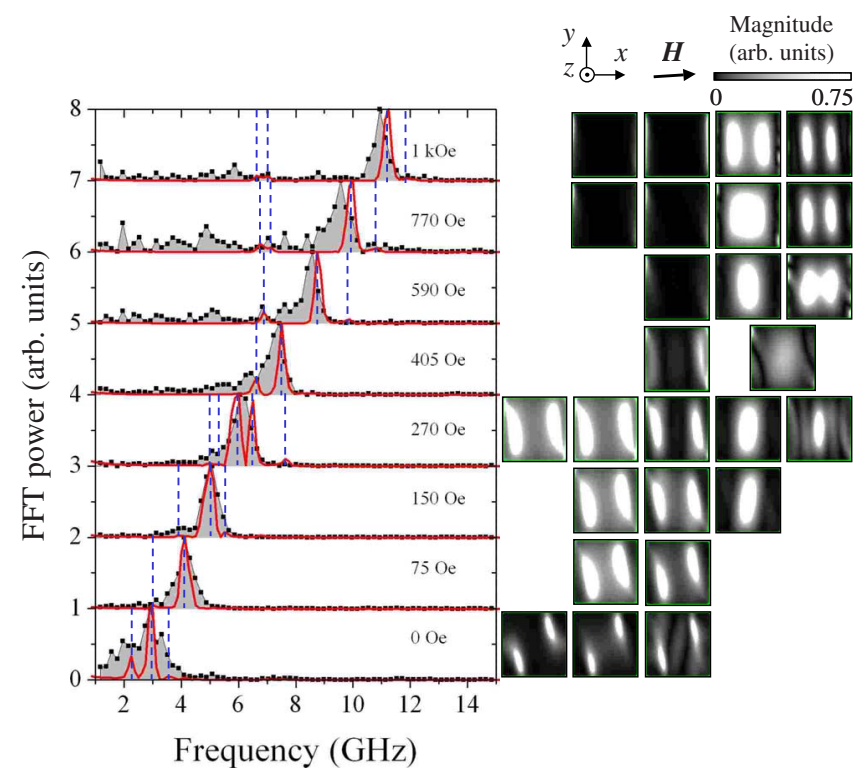

FIG. 9. (Color online) The dependence of the mode spectra and spatial character of the different modes upon the bias field is shown for the $428 \mathrm{~nm}$ element. The shaded spectra with symbols show the experimental data while the solid red line shows spectra obtained from micromagnetic simulations. The vertical dashed blue lines indicate the frequencies of the modes whose spatial character is shown in the images of the magnitude of the Fourier transform. The images are ordered in terms of increasing frequency.

lar element may be varied continuously by adjusting the bias field. Since the edge profile for a particular element is the same for all field values, it is advantageous to concentrate upon the field dependence of the precessional modes.

In Figs. 8-12, the dependence of the precessional mode spectra upon the bias magnetic field is shown for element sizes of $637,428,236,124$, and $70 \mathrm{~nm}$, respectively. For the element size of $637 \mathrm{~nm}$ (Fig. 8) the experimental and simulated spectra show a monotonic decrease in mode frequencies in the range of $12-2 \mathrm{GHz}$ as the bias field is reduced from $1 \mathrm{kOe}$ to $0 \mathrm{Oe}$. The experimental and simulated spectra show evidence of lower frequency modes at bias fields of 770 Oe and below, albeit with varying spectral power. The Fourier images in the right-hand panel again show the spatial character of the simulated modes. The vertical blue dashed lines indicate the frequencies of the spectral peaks to which the Fourier images correspond. The images reveal that the mode with the largest spectral power between bias fields of 1 $\mathrm{kOe}$ and $150 \mathrm{Oe}$ is a center mode. At 75 Oe the two detached-edge modes possess greater spectral power than the center mode. At 0 Oe the spectral power of the center mode vanishes and the dominant excited modes are two detachededge modes. At a bias field of 150 Oe, we observed a fielddependent crossover of the spatial character of the modes in the simulations. The experimental spectra do not clearly reveal the crossover; however they do reveal that more than one mode is present. It is also interesting to note that modes with backward-volume-type character, with a greater number of nodes and hence a greater wave number, have higher frequency than the quasiuniform mode. Such modes have previously been reported in Ref. 24. In Ref. 24 a very small 


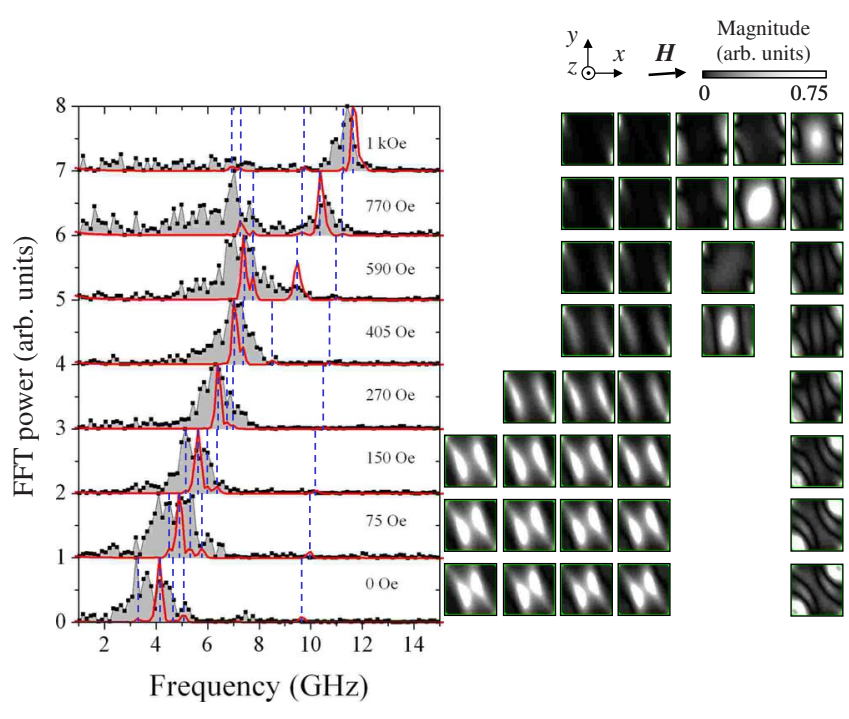

FIG. 10. (Color online) The dependence of the mode spectra and spatial character of the different modes upon the bias field is shown for the $236 \mathrm{~nm}$ element. The shaded spectra with symbols show the experimental data while the solid red line shows spectra obtained from micromagnetic simulations. The vertical dashed blue lines indicate the frequencies of the modes whose spatial character is shown in the images of the magnitude of the Fourier transform. The images are ordered in terms of increasing frequency.

damping parameter of 0.00001 was used in the micromagnetic simulations, which allow the quasiuniform and backward-volume-type modes to be resolved. Here a more realistic damping parameter of 0.01 was used and the linewidth of the two types of mode were overlapped. Therefore, the individual modes cannot be completely resolved in the Fourier imaging analysis.

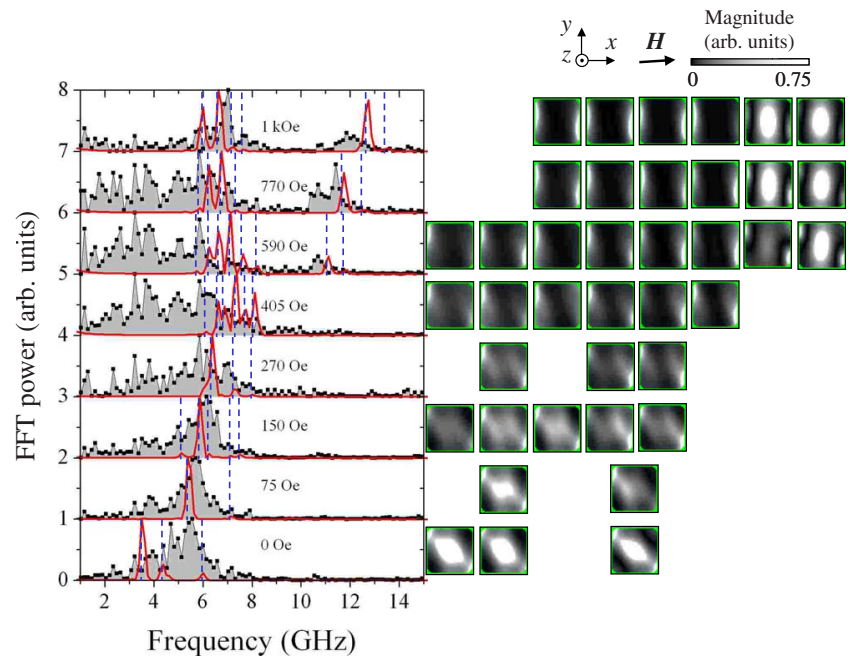

FIG. 11. (Color online) The dependence of the mode spectra and spatial character of the different modes upon the bias field is shown for the $124 \mathrm{~nm}$ element. The shaded spectra with symbols show the experimental data while the solid red line shows spectra obtained from micromagnetic simulations. The vertical dashed blue lines indicate the frequencies of the modes whose spatial character is shown in the images of the magnitude of the Fourier transform. The images are ordered in terms of increasing frequency.

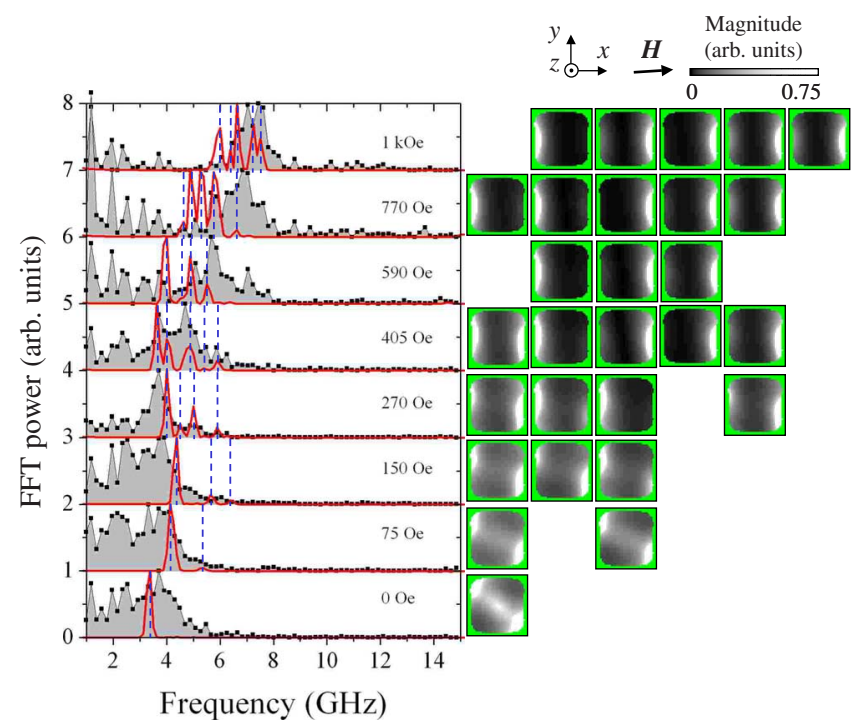

FIG. 12. (Color online) The dependence of the mode spectra and spatial character of the different modes upon the bias field is shown for the $70 \mathrm{~nm}$ element. The shaded spectra with symbols show the experimental data while the solid red line shows spectra obtained from micromagnetic simulations. The vertical dashed blue lines indicate the frequencies of the modes whose spatial character is shown in the images of the magnitude of the Fourier transform. The images are ordered in terms of increasing frequency.

For the element size of $428 \mathrm{~nm}$ (Fig. 9), the field dependence of the mode character is similar to that of the $637 \mathrm{~nm}$ element. Again the experimental and simulated spectra show a monotonic decrease in mode frequencies in the range of $12-2 \mathrm{GHz}$ as the bias field is reduced from $1 \mathrm{kOe}$ to $0 \mathrm{Oe}$. At a bias field of $1 \mathrm{kOe}, 770 \mathrm{Oe}$, and $590 \mathrm{Oe}$, evidence of lower frequency edge modes can be seen in both the experimental and simulated spectra. However, the spectral power of these modes is small and difficult to distinguish from noise and spectral peaks due to the pulsed field background.

One criterion to help identify a mode is to observe a peak with gradually changing frequency in successive spectra. While there might appear to be modes at $\sim 6 \mathrm{GHz}$ at $1 \mathrm{kOe}$, $\sim 5 \mathrm{GHz}$ at $770 \mathrm{Oe}$, and at $590 \mathrm{Oe}$ with spectral power $\sim 20 \%-40 \%$ of that of the main spectral peak (and approximately twice as large as the background level), there is no clear field dependence. On the other hand, the simulated spectra also reveal modes with small spectral power at $\sim 6.5-7 \mathrm{GHz}$ that show little field dependence. Therefore, it is difficult to distinguish modes with low spectral power from noise. However, peaks due to noise in the experimental spectra seem to be formed from very few data points (e.g., at $2 \mathrm{GHz}$ at $1 \mathrm{kOe}, 770 \mathrm{Oe}$, and $590 \mathrm{Oe}$ ) and have no field dependence.

As previously discussed, at $1 \mathrm{kOe}$, interference between the high-frequency backward-volume-type mode and the center mode leads to a Fourier image that is nonuniform at the center of the element with two antinodes. This composite center mode continues to have the largest spectral power until the bias field is reduced to 270 Oe, where a detachededge mode becomes the mode with largest spectral power. At 150 Oe the spectral power of the center mode is greatly 
reduced and the detached-edge modes dominate the dynamical response as the bias field is reduced further. From the simulations we see that the field-dependent crossover of mode spatial character has taken place at a higher bias field value of 270 Oe.

For the element size of $236 \mathrm{~nm}$ (Fig. 10), a crossover from a center mode to a detached-edge mode occurs between bias fields of 770 and 590 Oe. The experimental spectra show that the lower frequency edge mode has greater spectral power at 770 Oe while the simulated spectra suggest that the edge mode becomes dominant at 590 Oe. As the bias field is reduced from $1 \mathrm{kOe}$ to $590 \mathrm{Oe}$, the lower frequency edge modes do not exhibit a monotonic decrease in frequency but instead increase in frequency by about $0.5 \mathrm{GHz}$. As for the 637 and $428 \mathrm{~nm}$ elements, the frequency of the center mode of the $236 \mathrm{~nm}$ element exhibits a monotonic decrease as the bias field is reduced before the mode amplitude is greatly attenuated at $270 \mathrm{Oe}$. Also, between bias field values of $1 \mathrm{kOe}$ and $405 \mathrm{Oe}$, the Fourier images reveal that the center mode is nonuniform. At $1 \mathrm{kOe}$, the mode at 11.7 $\mathrm{GHz}$ has a similar character to the quasiuniform mode identified in similar elements in Ref. 24. However, as the bias field is reduced below $1 \mathrm{kOe}$, the magnetization becomes more nonuniform (Fig. 6) and interelement magnetostatic interactions increase. Therefore, the mode character departs significantly from that seen in Ref. 24 for noninteracting elements with uniform static magnetization at $2 \mathrm{kOe}$. At all bias field values the simulated spectra and Fourier images reveal a nonuniform mode between 9.6 and $10.3 \mathrm{GHz}$. Similarly for bias field values of $1 \mathrm{kOe}$ to $590 \mathrm{Oe}$, a nonuniform mode exists between 9.8 and $9.5 \mathrm{GHz}$. Between $1 \mathrm{kOe}$ and 590 Oe at least one of the nonuniform modes has a similar frequency to the center mode. As seen for the 637 and 428 nm elements, it is not possible to completely resolve modes with similar frequencies since the spectral lines have a finite linewidth. At 590 Oe the mode with frequency of about 9.8 $\mathrm{GHz}$ at 770 Oe seems to merge with the center mode spectral peak. The corresponding Fourier image reveals significant nonuniformity of the high amplitude region at the center of the element. At 590 Oe the experimental spectral peak is very broad and is accompanied by two modes of similar frequency in the simulated spectrum. The inhomogeneous broadening is again attributed to the random nature of defects and irregularities at the edges of the elements, which causes dispersion of the frequency of the excited modes near the element edges due to variations in the local effective field and pinning of the magnetization. The Fourier images corresponding to simulated spectra for bias fields of 590 Oe and below reveal that the modes of different frequency are in fact weakly associated with the element edges. Finally, the simulated spectra reveal that for all bias field values lower frequency modes with different frequency possess very similar, if not the same, spatial character, which is the result of collective-type excitations across the $3 \times 3$ array (discussed later).

For the element size of $124 \mathrm{~nm}$ (Fig. 11), a crossover from a center mode to an edge mode occurs at $1 \mathrm{kOe}$. Between 1 $\mathrm{kOe}$ and 590 Oe the higher frequency edge mode is observed in both experimental and simulated spectra between 13 and $10 \mathrm{GHz}$. However, between bias fields of 770 and 270 Oe for frequencies below $8 \mathrm{GHz}$, the experimental spectra are very broad and noisy, making it difficult to identify any modes. Empirically, as the element size is reduced, the signal level decreases. The noise for all element sizes is similar; however the noise is greater at lower frequencies as a result of the pulsed field subtraction. As the bias field is reduced, the signal shifts into the noisy, low-frequency part of the spectrum. As a result the signals from the smaller elements (with lower signal level) at low bias field are more difficult to identify. Many of the peaks below $8 \mathrm{GHz}$ in the spectra for the 124 $\mathrm{nm}$ element have little or no field dependence (for example, at $3.25,3.75$, and $5 \mathrm{GHz}$ ) and may be attributed to the transient pulsed field background. The pulsed field background may affect less the spectra at lower bias field values since the amplitude of the magnetization precession is greater than that at higher field values. In line with previous discussion, smaller elements will be more susceptible to frequency dispersion and inhomogeneous broadening of spectra since the effects of edge defects and irregularities are more pronounced. Furthermore, inhomogeneous broadening becomes more significant as the element size is decreased since the response of more elements is sensed in the region of the focused optical probe. Therefore, it is expected that the spectra acquired from smaller elements at lower bias field are more complicated.

In light of the good agreement between the experimental and simulated spectra for the largest three elements, we use the simulated spectra to discuss the expected behavior of the $124 \mathrm{~nm}$ element. The simulated spectra show that there are many lower frequency modes (up to six) excited with different frequency and spectral power that would not be resolved in the experimental spectra due to inhomogeneous broadening. The simulated Fourier images reveal that the excited modes in smaller elements seem to be mostly edge-type modes, which again are more susceptible to edge conditions. The Fourier images reveal that between $1 \mathrm{kOe}$ and $590 \mathrm{Oe}$ there are two center modes with different frequency, which are the result of collective-type excitations (discussed later). Similarly, many of the lower frequency edge-type modes share the same spatial character but again with different frequency. Between 270 and 75 Oe the high amplitude regions of the edge-type modes have extended into the center of the element while at 0 Oe the high amplitude region has become detached from the element edges. This may be interpreted as being due to a change of the static magnetization configuration. In the simulated spectra a large change in frequency $(\sim 2 \mathrm{GHz})$ of the largest spectral peak is seen between 75 and $0 \mathrm{Oe}$ which is not apparent in the experimental spectra. Simulated images of the static magnetization (Fig. 6) reveal that the magnetization at the center of the element has become significantly canted away from the $x$ direction as the regions of nonuniform magnetization along edges perpendicular to the bias field have extended toward the center of the element.

For the element size of $70 \mathrm{~nm}$ (Fig. 12), a crossover of mode character is not observed when the bias field is reduced, suggesting that the crossover occurs at higher field values. As for the $124 \mathrm{~nm}$ element there is a significant contribution to the experimental spectra from the pulsed field background. For example, the peak at $2 \mathrm{GHz}$ for all bias field 


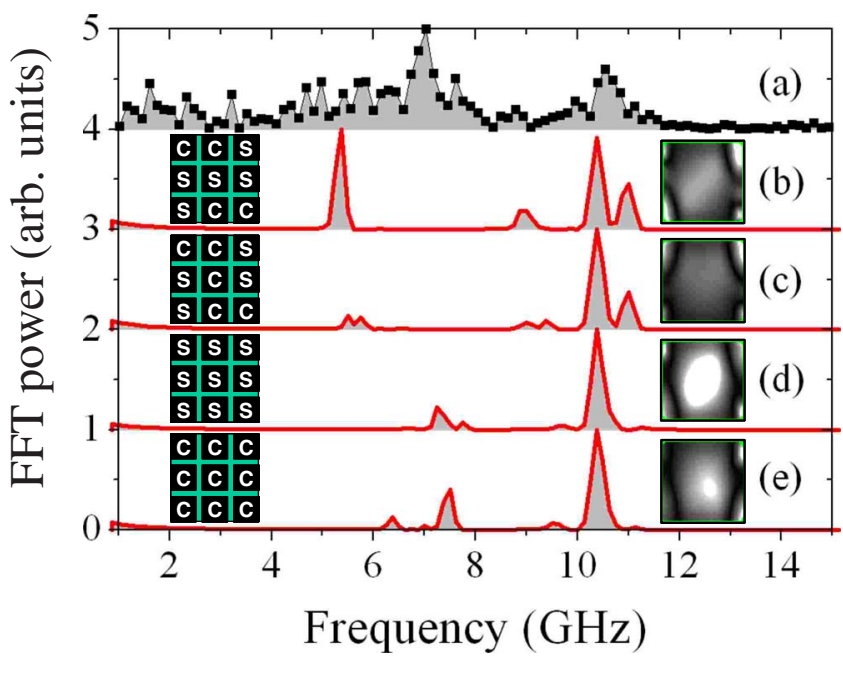

FIG. 13. (Color online) The experimental spectrum for the 236 $\mathrm{nm}$ element array at a bias field of 770 Oe is shown in (a). In (b)-(e) the simulated spectra derived from the average time-resolved response of the center element of $3 \times 3$ arrays are shown for different ground states. In (b) and (d) the simulated spectra correspond to the ground state shown for 770 Oe in Figs. 2(a) and 2(b), respectively. In (c) the ground state was similar to that in (b) except that the center element occupied the $C$ state. In (e) all elements occupied the $C$ state. For each case, illustrations and Fourier images (inset) show, respectively, the ground-state magnetization of the array and the center element spatial character for the mode at $10.4 \mathrm{GHz}$.

values is attributed to the pulsed field background. Again, in light of the good agreement between the experimental and simulated spectra for the largest three elements, we use the simulated spectra to discuss the expected behavior of the 70 $\mathrm{nm}$ element. The experimental and simulated spectra show that only lower frequency $(<8 \mathrm{GHz})$ modes are excited for bias fields of $1 \mathrm{kOe}$ and below. That is, the higher frequency branch of the center mode is not observed in either the experimental or simulated spectra. The corresponding Fourier images reveal that all modes excited between $1 \mathrm{kOe}$ and 150 Oe are edge-type modes. Again there is significant inhomogeneous broadening of the experimental spectra, which is supported by the presence of many modes with slightly different frequency in the simulated spectra. As for the $124 \mathrm{~nm}$ element, the high amplitude regions of the $70 \mathrm{~nm}$ element have extended into the center of the element between 75 and 0 Oe. In contrast with the $124 \mathrm{~nm}$ element, the simulated static magnetization reveals a transition from a nonuniform $S$ state to a more uniform leaf state between these bias field values. In addition an abrupt decrease in frequency $(\sim 1 \mathrm{GHz})$ is seen in the simulated spectra as a result of the transition. This is in agreement with the interpretation of the change in frequency for the $124 \mathrm{~nm}$ element between 75 and 0 Oe: the change in frequency is due to canting of the static magnetization at the center of the element.

Results of micromagnetic simulations on $3 \times 3$ arrays shown in Fig. 13 have revealed that the frequencies of edgetype modes are dependent upon the static state of the magnetization of the center element and the surrounding nearestneighbor elements. In Fig. 13(a) the experimental spectrum for the $236 \mathrm{~nm}$ element array at a bias field of 770 Oe is shown. In Figs. 13(b) and 13(d), the simulated spectra correspond to the response of the center element for which the ground states of the array at 770 Oe are shown in Figs. 2(a) and 2(b), respectively. The spectrum in Fig. 13(c) corresponds to a similar ground state to that shown for 770 Oe in Fig. 2(b) but the center element occupies the $C$ state instead. In the latter case the ground state was obtained by relaxing the magnetization from the state shown in Fig. 2(a) at $1 \mathrm{kOe}$ rather than relaxing from uniform saturation as in the case of Fig. 2(a) at 770 Oe. Illustrations of the ground state magnetization of the array are shown in the inset of Fig. 13 for each case.

Near the edges of the element perpendicular to the bias field, the canting of the magnetization away from the direction of the bias field is different for the $S$ and $C$ states (Fig. 2). For different static states the effective field near the element edge and the interelement interactions are different. Therefore, the frequency of modes excited in the edge regions can be different. The simulated spectra in Figs. 13(b) and 13(c) reveal splitting, a decrease in spectral power, and a small increase in frequency of the edge-type modes when the ground state of the center element is changed from an $S$ to a $C$ state. At the same time, the higher frequency modes, including the center mode at $10.4 \mathrm{GHz}$, are less affected. This is expected since the different edge conditions of the $C$ and $S$ states will mostly affect the frequency of the edge-type modes. The Fourier images in the inset of Fig. 13 reveal slight changes in the spatial character of the dynamic magnetization when either the static state of the center element [Figs. 13(b) and 13(c)] or the static state of the surrounding elements [e.g., Figs. 13(c) and 13(e)] is changed.

Comparison of the simulated spectra in Figs. 13(b) and 13(d), or Figs. 13(c) and 13(e) reveal the changes in the spectra due to interelement interactions when the static states of the nearest-neighbor elements are changed. In Figs. 13(b) and 13(d), the center element occupies the $S$ state. For the spectra shown in Fig. 13(b) the nearest-neighbor elements occupy $C$ and $S$ states while for the spectra shown in Fig. 13(d), all neighboring elements occupy the $S$ state. In Fig. 13(d) splitting of the edge-type mode is observed along with a significant increase in frequency of $\sim 2 \mathrm{GHz}$, which matches the frequency of the edge mode in the experimental spectra in Fig. 13(a). In contrast to the spectra in Figs. 13(b) and $13(\mathrm{c})$, the spectral power of the highest frequency mode at $11.25 \mathrm{GHz}$ is significantly reduced, which indicates that it too may be sensitive to interelement interactions. In the case when the $C$ state is induced in all the elements within the array [Fig. 13(e)], the spectrum is found to be very similar to that of the array in which all the elements occupy the $S$ state. However, as expected, the frequencies of the edge modes are affected to a greater extent than those of the center modes [Figs. 13(d) and 13(e)] as a result of the different static magnetization along the edges of the element perpendicular to the bias field for the $S$ and $C$ states.

So far the effect on the simulated spectra of rounded corners, interelement interaction, edge roughness, and static magnetization has been tested for the element size of 236 nm. For example, it is clear from Fig. 13 that the ground state of all elements in the array is important since the magnetostatic interactions between elements can modify the 


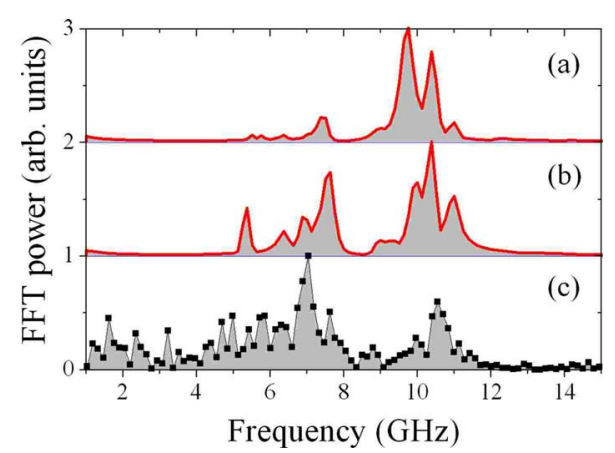

FIG. 14. (Color online) The averaged spectra for simulated elements that occupy the $C$ and $S$ states are shown in (a) and (b), respectively. In both cases the element size was $236 \mathrm{~nm}$ and the bias field was 770 Oe. The corresponding experimental spectrum is shown in (c). The spectrum in (a) is the average of equal contributions from the spectra shown in Figs. 7(d)-7(f), 13(c), and 13(e) while the spectra in (b) is the average of equal contributions from the spectra shown in Figs. 7(a)-7(c), 13(b), and 13(d).

spectra while Fig. 7 reveals that edge roughness is important, particularly if the simulated elements occupy the $S$ state. However the spectra obtained from experimentally acquired time-resolved Kerr signals are influenced by all of these conditions simultaneously. Therefore, in attempting to reproduce the detailed structure of the experimental spectra, it is necessary to combine the effects of these different conditions by averaging the corresponding spectra. Figures 14(a) and 14(b) show the averaged spectra for simulated elements that occupy the $C$ and $S$ states, respectively. In both cases the element size was $236 \mathrm{~nm}$ and the bias field was 770 Oe. The corresponding experimental spectrum is shown in Fig. 14(c). The spectrum in Fig. 14(a) is the average of equal contributions from the spectra shown in Figs. 7(d)-7(f), 13(c), and 13(e) while Fig. 14(b) is the average of equal contributions from the spectra shown in Figs. 7(a)-7(c), 13(b), and 13(d). Figures 14(a) and 14(b) reveal that the combination of edge roughness, interelement dipolar interactions, and different magnetic ground states of the $3 \times 3$ array significantly enhances the detailed structure of the spectra. Figures 14(a) and 14(b) show very different spectra for elements in the $C$ and $S$ states, respectively. In particular the edge (lower frequency) modes have a greater spectral power for the elements that occupy the $S$ state. Figure 14(c) shows that there remain significant differences between the simulated and experimental spectra. Indeed, by averaging the spectra for different simulated models, more structures are observed in the simulated spectra in Figs. 14(a) and 14(b). Some of the detailed structure of the experimental spectra may then be attributed to the dispersion of excited modes due to the edge roughness, interelement interactions, and different magnetic ground states. In addition, for elements of length of $236 \mathrm{~nm}$ or less, an ensemble of elements beneath the optical probe is measured. A total of five contributing spectra were combined to produce the spectra in Fig. 14, which is approximately the same number of elements (of length of $236 \mathrm{~nm}$ and separation of $77 \mathrm{~nm}$ ) sampled by the submicrometer optical probe in the experiment. As already discussed the slight variations in element edge roughness and magnetic ground state can

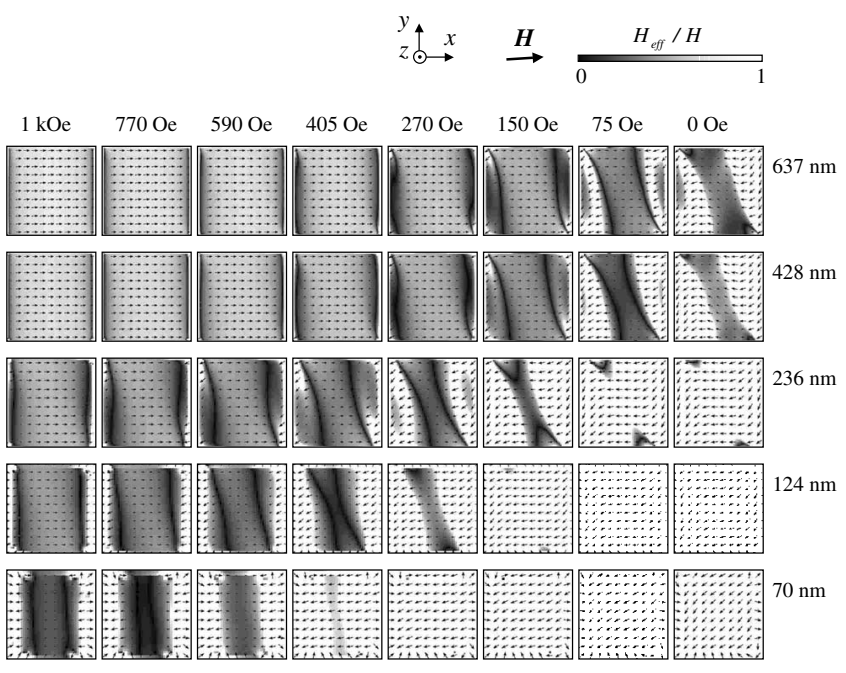

FIG. 15. The simulated total effective field within the center element of the $3 \times 3$ model arrays is shown. The effective field includes contributions from the applied, demagnetizing, anisotropy, and exchange fields. The grayscale represents the magnitude of the effective field normalized to the bias field except at remanence where the field was normalized to 75 Oe.

further enhance the broadening of the spectra.

The different contributing spectra that form the averaged spectra shown in Figs. 14(a) and 14(b) can be weighted to enhance the contribution of a particular effect, e.g., edge roughness, so that the simulated spectra are in better agreement with experimental spectra. However, in order to achieve better agreement, the weighting would be arbitrary and might not represent the contributions to the spectra measured experimentally. Therefore, here we conclude that a combination of the different conditions can lead to a simulated spectrum that is more representative of the spectra measured experimentally; however it is not possible to uniquely identify the weight of each contribution.

It is clear from the images in Fig. 6 that the static state of the magnetization becomes increasingly nonuniform as the bias field is decreased. In Fig. 15 images of the total effective field $\left(H_{\text {eff }}\right)$ corresponding to the magnetization configurations in Fig. 6 are shown. The effective field includes contributions from the applied, demagnetizing, anisotropy, and exchange fields. The grayscale represents the magnitude of the effective field normalized to the bias field. The images clearly show that a reduction in either the element size or the bias field can result in a nonuniform effective field. At $1 \mathrm{kOe}$, the effective field is nearly uniform at the center of all elements (light gray). For the 637 and $428 \mathrm{~nm}$ elements, regions of small internal field exist in narrow regions along the edges perpendicular to the applied field (black). As the element size is reduced from 236 to $70 \mathrm{~nm}$, the regions of small internal field move towards the center of the element and remain perpendicular to the bias field. Similarly, when the bias field is reduced from $1 \mathrm{kOe}$, the regions of small internal field within the 637 and $428 \mathrm{~nm}$ elements begin to move towards the center of the element at 405 Oe. However, at bias field values of 405 Oe and below, the effective field becomes nonuniform in both $x$ and $y$ directions. At 75 Oe regions of negative internal field can clearly be seen from the direction 


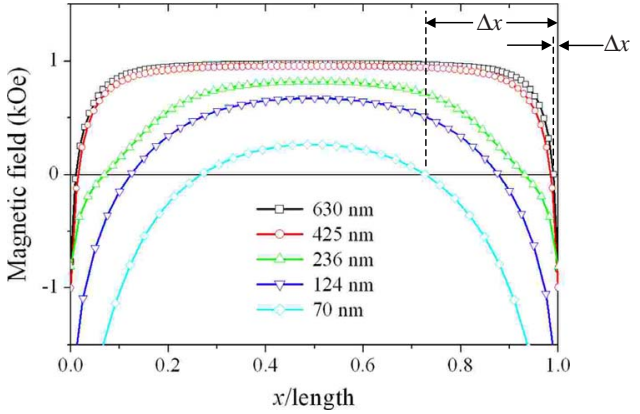

FIG. 16. (Color online) Cross sections of the simulated total effective field within the center element of the $3 \times 3$ model arrays at a bias field of $1 \mathrm{kOe}$. The sections show the $x$ component of the effective field. Regions of negative effective field exist for all element sizes. The width of the regions, labeled as $\Delta x$, is found to occupy a larger proportion of the total element length as the element size is reduced.

of the arrows. A similar evolution of the effective field is seen for the 236 and $124 \mathrm{~nm}$ elements as the bias field is reduced; however it seems that regions of small internal field are observed at bias field values of $1 \mathrm{kOe}$ and greater. At a bias field value (element size) of 0 (637 and $428 \mathrm{~nm}), 150$ $(236 \mathrm{~nm}), 270(124 \mathrm{~nm})$, and 590 Oe $(70 \mathrm{~nm})$, the effective field becomes negative throughout the majority of the element. The most significant contribution to the effective field is then the demagnetizing field, and the regions of small internal field (black) are expelled from the element near the edges parallel to the bias field.

In Fig. 16 cross sections of the effective-field images (shown in Fig. 15) for a bias field of 1 kOe are shown for each element size. The sections were taken parallel to the $x$ direction and through the center of the elements. The sections reveal that for all element sizes regions of negative effective field exist along the edges of the element perpendicular to the bias field. For the $637 \mathrm{~nm}$ element the negative effective field occupies very narrow regions with net width $2 \Delta x$ less than $2 \%$ of the total element length. In contrast, in the $70 \mathrm{~nm}$ element, the net width of the regions is $54 \%$ of the total element length.

For the 637, 236, and $70 \mathrm{~nm}$ elements, respectively, Figs. 17-19 show cross sections of (a) the effective-field images (shown in Fig. 15), and (b) the FFT magnitude of the dominant edge and center modes, where both appear (shown in Figs. 8, 10, and 12) for four different bias field values. Generally there are three regions of interest in the cross section of the effective field: (i) a region of zero or negative effective field adjacent to the edges of the element perpendicular to the bias field (edge region), (ii) a region of positive effective field at the center of the element (center region), and (iii) a region that connects the first two regions where the gradient of the effective field is large (gradient region).

For the $637 \mathrm{~nm}$ element, Fig. 17(a) reveals that there is a small increase in the width $2 \Delta x$ of the edge regions of about $4 \%$ of the element length as the bias field is reduced from 1 kOe to 270 Oe. At the same time, the magnitude of the effective field in the edge regions changes from about -750 to -250 Oe. At 75 Oe it can be seen that the width of the edge regions has increased significantly to about $56 \%$ of the

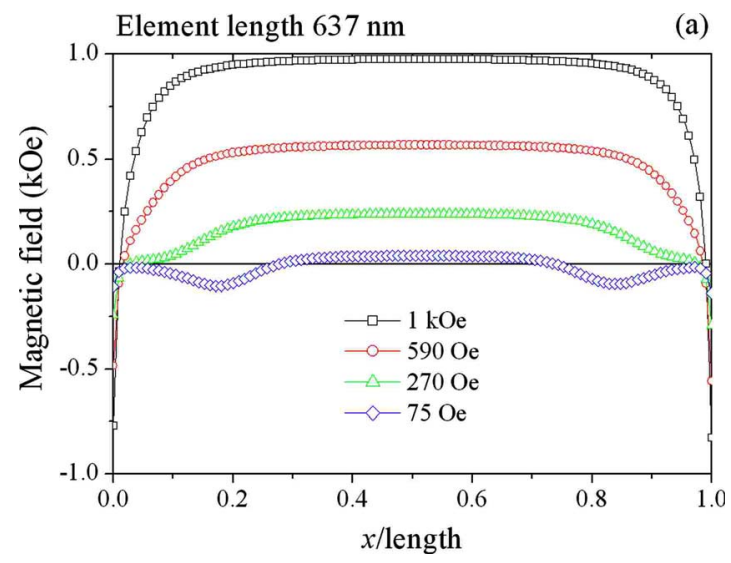

(b)

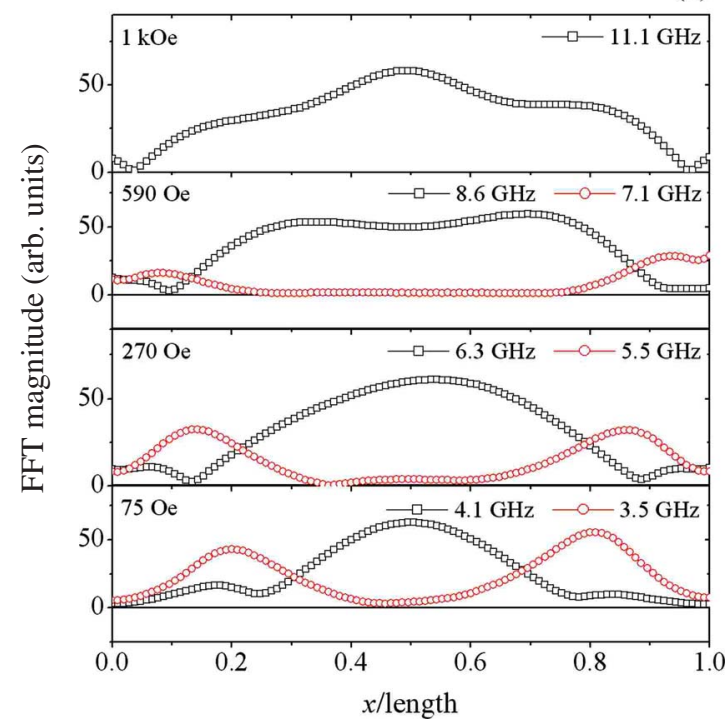

FIG. 17. (Color online) Cross sections are shown of (a) the $x$ component of the simulated total effective field and (b) the FFT magnitude within the center element of the $3 \times 3$ model array of $637 \mathrm{~nm}$ elements. The sections are shown for four different values of the bias field.

element length, and a maximum in the magnitude of the negative effective field has formed. Within these edge regions at $75 \mathrm{Oe}$, the magnitude of the negative effective field is larger than the positive effective field in the center region. As the bias field is reduced from $1 \mathrm{kOe}$ to $75 \mathrm{Oe}$, the effective field in the center region remains approximately equal to the bias field. Furthermore, the width of the gradient regions increases at the expense of that of the center region.

In Fig. 17(b) two types of modes are seen from the cross section of the FFT magnitude images: (i) a mode with large FFT magnitude at the center of the element (open black squares) and (ii) a mode with large FFT magnitude near the edges of the element perpendicular to the bias field (open red circles). It would be incorrect to characterize these modes in terms of strict localization to the center, edge, or gradient regions. For example, for a mode to be center (or edge) localized, one would expect the FFT magnitude to be equal to zero at the edges (or center) of the element. In Fig. 17(b) at $1 \mathrm{kOe}$ the $11.1 \mathrm{GHz}$ mode has two minima in the profile of the FFT magnitude near the element edges. As the bias field 


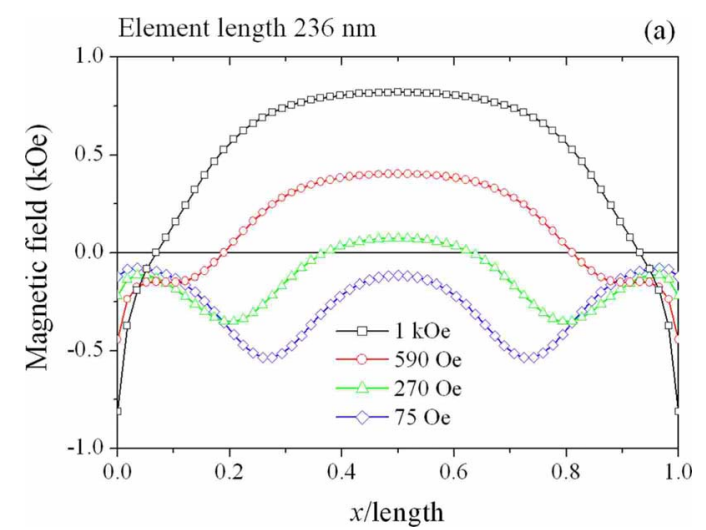

(b)

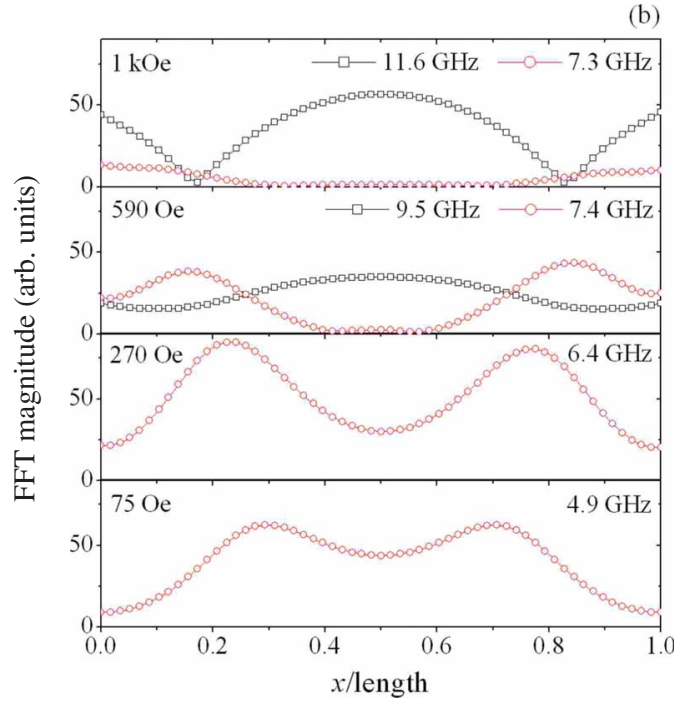

FIG. 18. (Color online) Cross sections are shown of (a) the $x$ component of the simulated total effective field and (b) the FFT magnitude within the center element of the $3 \times 3$ model array of $236 \mathrm{~nm}$ elements. The sections are shown for four different values of the bias field.

is reduced, the minima migrate from positions near the edge of the element at $1 \mathrm{kOe}$ toward the center of the element. At 590 Oe the lower frequency mode $(7.1 \mathrm{GHz})$ has two maxima in the profile of the FFT magnitude that correspond to the position of the two minima of the higher frequency $(8.6 \mathrm{GHz})$ mode. As the bias field is reduced these maxima also migrate toward the center of the element.

Features in the cross section of the FFT magnitude for both modes are correlated with those of the effective field in Fig. 17(a). In Ref. 11 for a transversely magnetized microscale stripe, a gradient region near the stripe edge was identified as the region of localization for low-frequency modes with edge-type spatial character. The edge region (zero-field and nonuniform magnetization) was assumed to reflect spin waves propagating from the center of the stripe toward these regions. Furthermore, a second turning point in the effective field was identified, above which only modes with an imaginary effective wave vector could exist. The region between these two boundaries was identified as the region of localization of the lowest frequency mode. Here, in smaller submicron nonellipsoidal elements, the lower frequency modes have large FFT magnitude in the gradient regions but are not
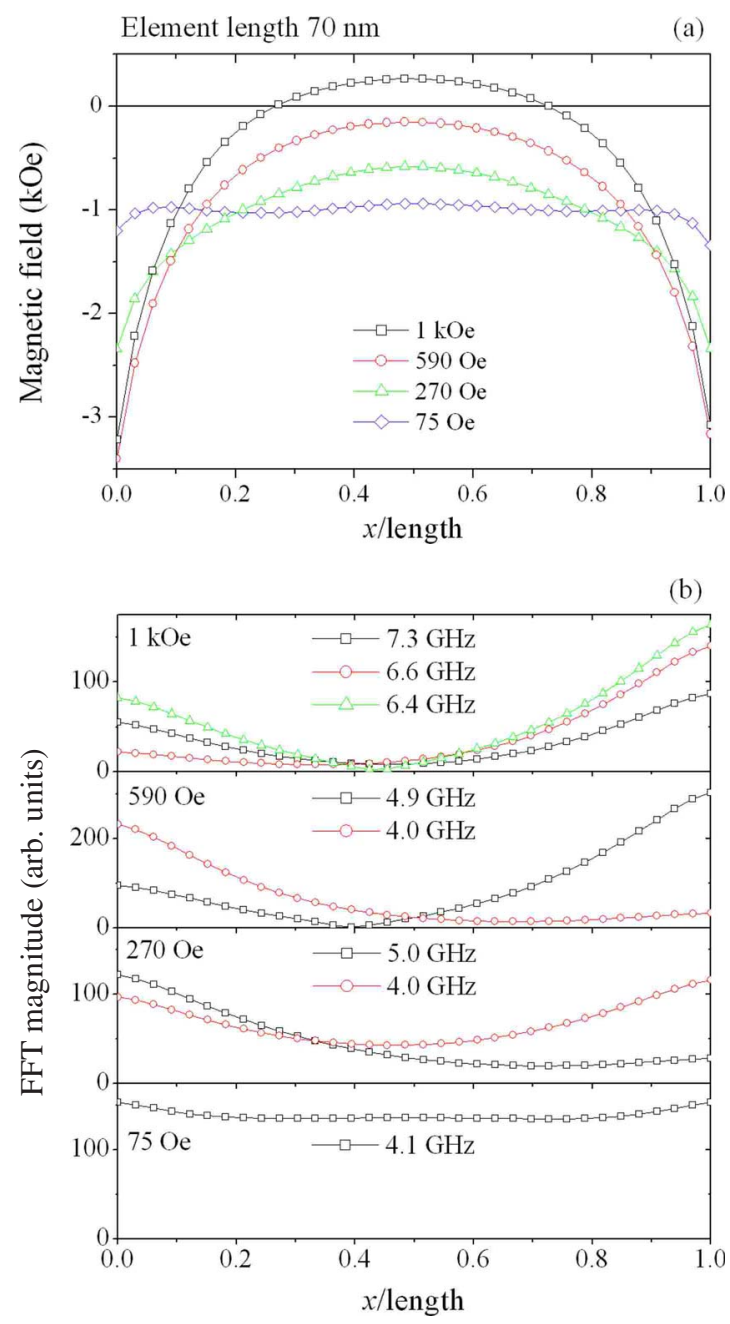

FIG. 19. (Color online) Cross sections are shown of (a) the $x$ component of the simulated total effective field and (b) the FFT magnitude within the center element of the $3 \times 3$ model array of 70 $\mathrm{nm}$ elements. The sections are shown for four different values of the bias field.

localized there. This is in agreement with Ref. 17 in which the spin-wave well model was extended to account for the nonuniform static state magnetization. Here it is found that the FFT magnitude extends across regions of positive and negative internal fields, as seen in Fig. 17(b) at 590 Oe. The region of large FFT magnitude associated with the higher frequency mode decreases in width as the bias field is decreased. At the same time, the FFT magnitude of the lower frequency mode increases near the edge of the elements and migrates toward the center of the element. However, while the effective field is positive in the center region, the FFT amplitude of the lower frequency mode in that region remains small.

For the $236 \mathrm{~nm}$ element at $590 \mathrm{Oe}$, the effective field [Fig. $18(\mathrm{a})]$ in the center region is positive and the FFT magnitude [Fig. 18(b)] of the lower frequency mode is small. However, at $75 \mathrm{Oe}$, the effective field is negative across the whole section of the element. The regions of high FFT magnitude of the lower frequency mode that were near the element edges now also extend across the whole element. It is clear 
from Fig. 18(a) that the effective field within the center region of the element is no longer similar to the bias field. Instead, the demagnetizing field contributes significantly to the effective field.

For the $70 \mathrm{~nm}$ element, the effective field [Fig. 19(a)] is only positive in the center region when the bias field is 1 $\mathrm{kOe}$, and is significantly less than the bias field value. As discussed earlier, modes from the higher frequency branch that were found occupying the center region of larger elements (Figs. 8-11) were not observed in the simulation of 70 $\mathrm{nm}$ elements at $1 \mathrm{kOe}$ (Fig. 12). Instead, at $1 \mathrm{kOe}$ the modes excited within the $70 \mathrm{~nm}$ element have large FFT magnitude in the edge regions [Fig. 19(b)], where the effective field is large $(\sim 3 \mathrm{kOe})$ and negative. In the center region where the effective field is positive, the FFT magnitude of the modes is small. Between the bias field values of 590 and 75 Oe, the large demagnetizing field results in a negative effective field across the whole section of the element. Figure 12 reveals a number of different nonuniform modes that tend to have maximum magnitude at the edges of the element. Figure 19(b) reveals that the FFT magnitude of these modes is nonzero throughout the element, with regions of large FFT magnitude correlated with regions of large negative internal field. Finally, at 75 Oe, Figs. 6 and 15 reveal that the $70 \mathrm{~nm}$ element no longer occupies the $S$ state but now occupies the leaf state. In this case the negative effective field is almost constant across the whole section of the element.

It is clear from Fig. 18(b) that the crossover observed in the spectra and Fourier images of Fig. 10 is the result of a change in the spatial character of the excited modes. The crossover is mediated by the changes in the effective field throughout the element as the bias field is decreased. However, since the modes have nonzero FFT magnitude throughout, the crossover cannot be simply described as a crossover from a center-localized to an edge-localized mode character. In a similar manner the change in the effective field that occurs as the element size is reduced (Fig. 15) can be used to explain the size dependent crossover of the spatial character of the modes seen in Fig. 5.

\section{COLLECTIVE MODES WITHIN ARRAYS}

In Ref. 18 the effect of interelement interactions upon high-frequency normal modes was modeled in $3 \times 3$ square arrays of circular Permalloy dots of thickness of $50 \mathrm{~nm}$, diameter of $200 \mathrm{~nm}$ and interdot separation ranging from 50 to $800 \mathrm{~nm}$. For an interelement separation greater than about $200 \mathrm{~nm}$, the high-frequency response was found to be successfully modeled by that of an individual element. For interelement separations less than $200 \mathrm{~nm}$, the magnetostatic interaction was found to significantly modify the mode character in the dots. Here, the interelement separation is less than $200 \mathrm{~nm}$ for all element sizes, and the separation is not varied. However, by varying the bias field the magnetostatic interaction between elements can be changed and investigated.

So far in this paper we have interpreted the experimental spectra by comparing them with the corresponding simulated spectra for the center element of $3 \times 3$ model arrays and then (a)

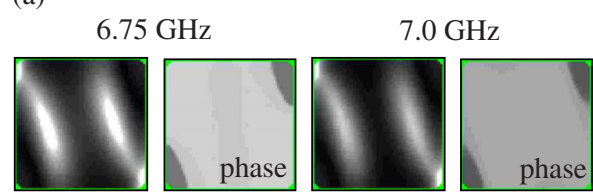

(b)

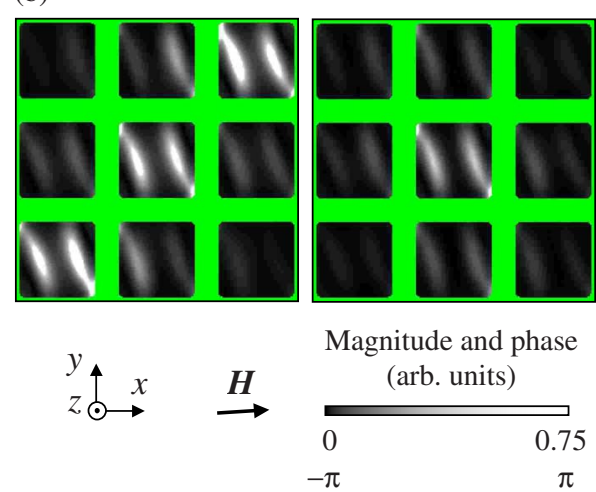

FIG. 20. (Color online) Simulated Fourier images of FFT magnitude and phase are shown in (a) for two modes with frequencies of 6.75 and $7.0 \mathrm{GHz}$ excited in the center element of a $3 \times 3$ array of $236 \mathrm{~nm}$ elements at a bias field of 270 Oe. In (b) the FFT magnitude is shown for all elements in the $3 \times 3$ array.

calculating the Fourier images of the spatial character for particular modes. For all element sizes, particularly for the lower frequency modes $(<8 \mathrm{GHz})$, the spatial character of modes excited in the center element are similar if not the same as other excited modes for a particular element size and bias field. We interpret this as evidence for collective modes within the $3 \times 3$ array. The simulated Fourier images of the center element reveal that the spatial character of the FFT magnitude and phase are similar for modes with different frequency. However, in these cases the spatial character of amplitude and phase across the $3 \times 3$ array were found to be different.

In Fig. 20(a), the FFT magnitude and phase are shown for two modes with frequencies of 6.75 and $7.0 \mathrm{GHz}$ excited in the center element of a $3 \times 3$ array of $236 \mathrm{~nm}$ elements. While there is a finite phase difference between the two modes, the variation in the phase within each image is similar. In Fig. 20(b) the FFT magnitude is shown for all elements in the $3 \times 3$ array. While the spatial character of the two modes is similar in the center element, the character of the modes across the array are very different, demonstrating that collective modes occur within the array. The excitation of collective modes with similar frequencies may manifest itself as linewidth broadening of the experimental spectra in Figs. 8-12 since the collective modes cannot be resolved experimentally. The Fourier images in Figs. 8-12 reveal that below the crossover field there are often several modes at a particular bias field with similar spatial character. Indeed, as the bias field is reduced, the static state of the magnetization becomes increasingly nonuniform and the magnetostatic interaction between elements increases. ${ }^{10}$ Therefore, below the crossover field a larger splitting of the frequencies of the collective modes is expected. This is supported by the increased linewidth observed experimentally at lower bias field values. 
By placing the magneto-optical probe at different positions in the array, one can expect to acquire a different response to the uniform pulsed magnetic field as a result of the spatial character of the collective modes. The most insightful method of investigating the spatial character of collective modes within arrays is to image these modes using timeresolved scanning Kerr microscopy. In order to excite and image a single collective mode, harmonic field excitation is required at the resonant frequency of the mode. In the present study this was not possible since the pulsed magnetic field was triggered using a photoconductive switch.

\section{SUMMARY}

We have performed TRSKM measurements upon arrays of square ferromagnetic nanoelements of different sizes and for a range of bias fields. The experimental results were compared to micromagnetic simulations of model arrays in order to understand nonuniform precessional dynamics within the elements. Experimentally, two branches of excited modes were observed to coexist above a particular bias field. Below the so-called crossover field, the higher frequency branch was observed to vanish.

Micromagnetic simulations and Fourier imaging revealed the spatial character of the two mode branches. The modes of the higher frequency branch were found to have high FFT amplitude at the center of the element in regions of positive effective field while modes of the lower frequency branch were found to have high FFT amplitude near the edges of the element perpendicular to the bias field. Cross sections of the simulated images of the effective field and FFT magnitude revealed that the crossover between the higher and lower frequency branches was mediated by the complicated evolution of the total effective field within the element. Below the crossover field the increase in the width of the edge region, which is of negative effective field, at the expense of the center region, which is of positive effective field, allowed the edge-type mode to extend over the entire element. The simulations revealed that the majority of the modes were delocalized with nonzero FFT magnitude throughout the element. The regions of large FFT magnitude were found to exist in the gradient region of the total internal field in agreement with Ref. 17 in which the spin-wave well model was ex- tended for nonuniform static state magnetization. The delocalized nature of the excited modes seems to be an intrinsic property of submicrometer and nanoscale nonellipsoidal elements for which the mode spatial character was found to be correlated with features of the effective field and the static state magnetization.

The simulations revealed that the frequency of modes from the lower frequency branch were very sensitive to the element edge roughness via the static state magnetization, the interelement interactions of elements within arrays, and the particular static magnetization state ( $S$ or $C$ state) of all the elements within the model array. These effects were found to significantly enhance the detailed structure of the spectra in $236 \mathrm{~nm}$ elements, which may account for the significant broadening and unresolved background of the experimental spectra. Since below the crossover field the static magnetization state was shown to be nonuniform, increased magnetostatic interaction is expected between the elements within the array. The simulated spectra and Fourier images revealed that many excited modes have similar spatial character within the center element. However, inspection of the entire array revealed the existence of collective modes, where the FFT amplitude in the elements surrounding the center element was considerably different for two such modes. The excitation of collective modes with similar spatial character in the center element but different frequency may manifest itself as an increased linewidth observed in the experimental spectra below the crossover field.

Finally, the results presented in this experimental paper may be useful for the development of a thorough analytical theory of nonuniform modes within magnetic nanoelements with nonuniform static magnetization and total effective field. Detailed knowledge of the mode character of square nanoelements and their static magnetization state is also essential for data storage applications in the finite field regime.

\section{ACKNOWLEDGMENTS}

We acknowledge W. A. Murray of the School of Physics, University of Exeter, UK, for the acquisition of SEM images of the arrays. Financial support for this work was provided by the UK Engineering and Physical Sciences Research Council (EPSRC) and the New Energy and Industrial Technology Development Organization (NEDO).
${ }^{1}$ J. R. Childress and R. E. Fontana, Jr., C. R. Phys. 6, 997 (2005).

${ }^{2}$ R. C. Sousa and I. L. Prejbeanu, C. R. Phys. 6, 1013 (2005).

${ }^{3}$ J. Z. Sun, IBM J. Res. Dev. 50, 8 (2006).

${ }^{4}$ S. Tamaru, J. A. Bain, R. J. M. van de Veerdonk, T. M. Crawford, M. Covington, and M. H. Kryder, Phys. Rev. B 70, 104416 (2004).

${ }^{5}$ M. R. Freeman, R. R. Ruf, and R. J. Gambino, IEEE Trans. Magn. 27, 4840 (1991).

${ }^{6}$ A. V. Kimel, A. Kirilyuk, F. Hansteen, R. V. Pisarev, and Th. Rasing, J. Phys.: Condens. Matter 19, 043201 (2007).

${ }^{7}$ M. Tsoi, A. G. M. Jansen, J. Bass, W. C. Chiang, M. Seck,
V. Tsoi, and P. Wyder, Phys. Rev. Lett. 80, 4281 (1998).

${ }^{8}$ A. G. Gurevich and G. A. Melkov, Magnetization Oscillations and Waves (Chemical Rubber, New York, 1996).

${ }^{9}$ P. H. Bryant, J. F. Smyth, S. Schultz, and D. R. Fredkin, Phys. Rev. B 47, 11255 (1993).

${ }^{10}$ S. O. Demokritov, B. Hillebrands, and A. N. Slavin, Phys. Rep. 348, 441 (2001), and references therein.

${ }^{11}$ J. Jorzick, S. O. Demokritov, B. Hillebrands, M. Bailleul, C. Fermon, K. Y. Guslienko, A. N. Slavin, D. V. Berkov, and N. L. Gorn, Phys. Rev. Lett. 88, 047204 (2002).

${ }^{12}$ J. P. Park, P. Eames, D. M. Engebretson, J. Berezovsky, and P. A. 
Crowell, Phys. Rev. Lett. 89, 277201 (2002).

${ }^{13}$ S. M. Chérif, Y. Roussigné, C. Dugautier, and P. Moch, J. Magn. Magn. Mater. 242-245, 591 (2002).

${ }^{14}$ G. Gubbiotti, M. Conti, G. Carlotti, P. Candeloro, E. Di Fabrizio, K. Y. Guslienko, A. Andre, C. Bayer, and A. N. Slavin, J. Phys.: Condens. Matter 16, 7709 (2004).

${ }^{15}$ V. V. Kruglyak, A. Barman, R. J. Hicken, J. R. Childress, and J. A. Katine, J. Appl. Phys. 97, 10A706 (2005).

${ }^{16}$ V. V. Kruglyak, A. Barman, R. J. Hicken, J. R. Childress, and J. A. Katine, Phys. Rev. B 71, 220409(R) (2005).

${ }^{17}$ M. Bailleul, R. Höllinger, and C. Fermon, Phys. Rev. B 73, 104424 (2006).

${ }^{18}$ G. Gubbiotti, M. Madami, S. Tacchi, G. Carlotti, and T. Okuno, J. Appl. Phys. 99, 08 C701 (2006).

${ }^{19}$ V. V. Kruglyak, P. S. Keatley, R. J. Hicken, J. R. Childress, and J. A. Katine, J. Appl. Phys. 99, 08F306 (2006).

${ }^{20}$ C. Bayer, J. Jorzick, S. O. Demokritov, A. N. Slavin, K. Y. Guslienko, D. V. Berkov, N. L. Gorn, M. P. Kostylev, and B. Hillebrands, in Spin Dynamics in Confined Structures III, Topics in Applied Physics Vol. 101 (Springer, Berlin, Heidelberg, 2006), p. 57, and references therein.

${ }^{21}$ A. Barman, S. Q. Wang, J. D. Maas, A. R. Hawkins, S. Kwon, A. Liddle, J. Bokor, and H. Schmidt, Nano Lett. 6, 2939 (2006).

${ }^{22}$ V. V. Kruglyak, P. S. Keatley, R. J. Hicken, J. R. Childress, and J. A. Katine, Phys. Rev. B 75, 024407 (2007).

${ }^{23}$ F. Giesen, J. Podbielski, B. Botters, and D. Gründler, Phys. Rev. B 75, 184428 (2007).

${ }^{24}$ G. Gubbiotti, M. Madami, S. Tacchi, G. Carlotti, A. O. Adeyeye, S. Goolaup, N. Singh, and A. N. Slavin, J. Magn. Magn. Mater. 316, e338 (2007)

${ }^{25}$ M. L. Schneider, J. M. Shaw, A. B. Kos, Th. Gerrits, T. J. Silva, and R. D. McMichael, J. Appl. Phys. 102, 103909 (2007).

${ }^{26}$ M. R. Freeman, W. K. Hiebert, and A. Stankiewicz, J. Appl. Phys. 83, 6217 (1998).
${ }^{27}$ W. K. Hiebert, G. E. Ballentine, and M. R. Freeman, Phys. Rev. B 65, 140404(R) (2002).

${ }^{28}$ A. Barman, V. V. Kruglyak, R. J. Hicken, A. Kundrotaite, and M. Rahman, Appl. Phys. Lett. 82, 3065 (2003).

${ }^{29}$ A. Barman, V. V. Kruglyak, R. J. Hicken, J. M. Rowe, A. Kundrotaite, J. Scott, and M. Rahman, Phys. Rev. B 69, 174426 (2004).

${ }^{30}$ M. Buess, R. Höllinger, T. Haug, K. Perzlmaier, U. Krey, D. Pescia, M. R. Scheinfein, D. Weiss, and C. H. Back, Phys. Rev. Lett. 93, 077207 (2004).

${ }^{31}$ C. H. Back, D. Pescia, and M. Buess, in Spin Dynamics in Confined Structures III, Topics in Applied Physics Vol. 101 (Springer, Berlin, Heidelberg, 2006), p. 137, and references therein.

${ }^{32}$ B. C. Choi, J. Rudge, M. R. Freeman, Y. K. Hong, and Q. F. Xiao, IEEE Trans. Magn. 43, 2 (2007).

${ }^{33}$ For example, see the list of works citing M. J. Donohue and D. G. Porter, OOMMF Users Guide, Version 1.0, Interagency Report No. NISTIR 6376 (National Institute of Standards and Technology, Gaithersburg, MD, 1999).

${ }^{34}$ J. I. Martín, J. Nogués, K. Liu, J. L. Vicent, and I. K. Schuller, J. Magn. Magn. Mater. 256, 449 (2003), and references therein.

${ }^{35}$ L. Giovannini, F. Montoncello, and F. Nizzoli, Phys. Rev. B 75, 024416 (2007).

${ }^{36}$ P. S. Keatley, V. V. Kruglyak, R. J. Hicken, J. R. Childress, and J. A. Katine, J. Magn. Magn. Mater. 306, 298 (2006).

${ }^{37}$ P. S. Keatley, V. V. Kruglyak, A. Barman, S. Ladak, R. J. Hicken, J. Scott, and M. Rahman, J. Appl. Phys. 97, 10R304 (2005).

${ }^{38}$ M. J. Donohue and D. G. Porter, OOMMF Users Guide, Version 1.0, NISTIR 6376, 1999 (National Institute of Standards and Technology, Gaithersburg, MD, 1999).

${ }^{39}$ O. Fruchart and A. Thiaville, C. R. Phys. 6, 921 (2005). 